\title{
Spinal Interneuron Axons Spontaneously Regenerate after Spinal Cord Injury in the Adult Feline
}

\author{
Keith K. Fenrich and P. Ken Rose \\ Canadian Institute for Health Research Group in Sensory-Motor Integration, Department of Physiology, Center for Neuroscience, Queen's University, \\ Kingston, Ontario, Canada K7L 3N6
}

It is well established that long, descending axons of the adult mammalian spinal cord do not regenerate after a spinal cord injury (SCI). These axons do not regenerate because they do not mount an adequate regenerative response and growth is inhibited at the injury site by growth cone collapsing molecules, such as chondroitin sulfate proteoglycans (CSPGs). However, whether axons of axotomized spinal interneurons regenerate through the inhibitory environment of an SCI site remains unknown. Here, we show that cut axons from adult mammalian spinal interneurons can regenerate through an SCI site and form new synaptic connections in vivo. Using morphological and immunohistochemical analyses, we found that after a midsagittal transection of the adult feline spinal cord, axons of propriospinal commissural interneurons can grow across the lesion despite a close proximity of their growth cones to CSPGs. Furthermore, using immunohistochemical and electrophysiological analyses, we found that the regenerated axons conduct action potentials and form functional synaptic connections with motoneurons, thus providing new circuits that cross the transected commissures. Our results show that interneurons of the adult mammalian spinal cord are capable of spontaneous regeneration after injury and suggest that elucidating the mechanisms that allow these axons to regenerate may lead to useful new therapeutic strategies for restoring function after injury to the adult CNS.

\section{Introduction}

After injury to the nervous system, functional regeneration occurs when cut axons form growth cones, which elongate and navigate through or around the injury site, and form functional synaptic connections with appropriate targets. However, it is widely accepted that axons of the adult mammalian CNS are not capable of spontaneous functional regeneration after injury (Ramon y Cajal, 1959; Yiu and He, 2006). Regeneration fails partly because cut CNS axons do not typically acquire the appropriate proteins for growth cone formation and extension (Plunet et al., 2002). In addition, the few axons that do form growth cones are faced with the formidable barrier of navigating through the glial scar at the injury site. Glial scars contain growth inhibitory molecules such as chondroitin sulfate proteoglycans (CSPGs), myelin-derived inhibitory proteins, and repulsive guidance molecules (Silver and Miller, 2004; Harel and Strittmatter, 2006; Yiu and $\mathrm{He}, 2006)$.

Although the poor regenerative response and inhibitory effects of the injured CNS are formidable barriers, they can be overcome. For example, conditioning stimuli enhance central regeneration of sensory axons (Richardson and Issa, 1984; Neumann

\footnotetext{
Received Feb. 22, 2009; revised Aug. 4, 2009; accepted Aug. 20, 2009.

This work was supported by the Canadian Institutes for Health Research (MOP-79299). K.K.F. was supported by a Trevor C. Holland Fellowship, a Dr. Robert John Wilson Fellowship, and an Ontario Graduate Scholarship. We thank Rebecca Cranham for help with animal care, surgeries, and data acquisition; and Monica Neuber-Hess for help in conducting the experiments. We are most grateful to Steven Montague, Anirhuda Garg, and Laura Gedge for constructive and helpful feedback during revisions of this manuscript.

Correspondence should be addressed to Keith K. Fenrich, Department of Physiology, Queen's University, Kingston, Ontario, Canada, K7L 3N6. E-mail: keith@biomed.queensu.ca.

D0I:10.1523/JNEUROSCI.0897-09.2009

Copyright $\odot 2009$ Society for Neuroscience ～0270-6474/09/2912145-14\$15.00/0
}

and Woolf, 1999; Lu et al., 2004). Also, cleaving the active regions of inhibitory molecules (Bradbury et al., 2002) or using antibodies to block their action (Schnell and Schwab, 1990; Bregman et al., 1995) promotes regeneration. These studies show that certain classes of CNS neurons are capable of regenerating axons through or around a CNS injury site, but only with the aid of interventions that promote regeneration.

The goal of this study was to reexamine the ability of transected axons of CNS neurons to form functional connections in the absence of therapeutic interventions. This reexamination was based on observations of axons arising from spinal interneurons. Previous studies have shown that spinal interneurons survive well after axotomy caused by contusion spinal cord injury (SCI) (Conta and Stelzner, 2004) and, after midsagittal spinal transactions, axotomized spinal interneurons develop axon-like processes that cross the midline (Fenrich et al., 2007). In addition, the axons of spinal interneurons also have a remarkable capacity for the formation of new collateral branches that circumvent injury sites after partial SCIs (Bareyre et al., 2004; Courtine et al., 2008). However, although these studies speak to the abilities of spinal interneuron axons to sprout after SCI, they do not directly examine whether spinal interneuron axons are capable of spontaneous functional regeneration through an injury site. We therefore asked two fundamental questions: (1) Do spinal interneuron axons regenerate through an inhibitory environment in the absence of therapeutic interventions? and, if yes, (2) Do these regenerated axons form functional synaptic connections?

We examined propriospinal commissural interneurons (PCIs) and found that axotomized PCI axons regenerate through SCI lesion sites that contain dense deposits of inhibitory CSPGs. In 
addition, regenerated PCI axons were found to evoke inhibitory or excitatory postsynaptic potentials (PSPs) in motoneurons that typically receive input from PCIs. Our findings reveal, to our knowledge for the first time, that axons of spinal interneurons are capable of spontaneous functional regeneration after SCI.

\section{Materials and Methods}

\section{Surgeries}

All surgical and animal care protocols for these experiments were approved by the Queen's University Animal Care Committee and were consistent with the guidelines established by the Canadian Council of Animal Care. All surgical procedures were performed on adult cats $(>11$ months of age) under deep general anesthesia using either isoflurane (recovery experiments) or sodium pentobarbital (terminal experiments). Surgical procedures, peripheral nerve stimulation protocols, and postoperative care procedures were identical to those used in previous experiments (Fenrich et al., 2007). Briefly, for the recovery surgeries, the animal was anesthetized, and the dorsal C2 and C3 vertebrae were exposed and removed to access the $\mathrm{C} 3$ segment. The animal was suspended from a stereotaxic frame, the $\mathrm{C} 3$ nerves innervating the dorsal neck muscles biventer cervicis and complexus (BCCM) were isolated and mounted on stimulating electrodes, and the dura was resected to expose the dorsal surface of the spinal cord. We located the spinal midline in two ways. First, we recorded from antidromically activated BCCM motoneurons on both sides of the spinal cord. The point midway between the BCCM motoneuron pools was used as a marker for the position of the ventral midline. Second, we removed the arachnoid and pia matter to expose the dorsal median sulcus. The sulcus was used as a marker for the dorsal midline of the spinal cord. In nearly all experiments, the positions of the markers for the dorsal and ventral midlines were identical, and the scalpel blade was aligned to the center of the dorsal medial sulcus. However, on the occasions in which the positions of the markers for the dorsal and ventral midlines did not match (i.e., the spinal cord was slightly twisted), the point midway between the two was taken as the midline. We confirmed that all lesions transected the midline through the central canal after the terminal experiments (see below). The lesion protocol has previously been described in detail (Fenrich et al., 2007) and was performed using a specially designed double-edged scalpel blade that was mounted to a motorized microdrive. Briefly, the knife was inserted to a depth of at least $5 \mathrm{~mm}$ (the most ventral aspect of the ventral horns is $\sim 4 \mathrm{~mm}$ below the dorsal surface of the spinal cord at C3) (supplemental Fig. 1, available at www.jneurosci.org as supplemental material). The knife was advanced rostrally for a distance of 4 or $6 \mathrm{~mm}$, returned to its starting position, and advanced rostrally again for a distance of 4 or $6 \mathrm{~mm}$. Most of the lesions were $4 \mathrm{~mm}$ long. In one experiment in which the intended length of the lesion was $4 \mathrm{~mm}$, the blade was moved only $3.5 \mathrm{~mm}$ rostrocaudally because of a shorter laminectomy. Two animals received $6 \mathrm{~mm}$ lesions.

Animals were divided into three groups based on the time between lesion and terminal experiments; these groups were called " $0 \mathrm{~d}$," "7 d," and " $56-72 \mathrm{~d}$ " experiments. The $0 \mathrm{~d}$ animals were control experiments, and we proceeded to the terminal experiment immediately after the spinal lesion. Postoperative pain was controlled with buprenorphine $(0.005-0.01 \mathrm{mg} / \mathrm{kg}$, s.c.; every $6 \mathrm{~h}$ or as required) and/or Metacam (1 drop orally; per day as required). For the terminal experiment, the animals were anesthetized, the dorsal surface of the spinal cord was exposed from $\mathrm{C} 1$ to $\mathrm{C} 5$, and the cat was suspended in a stereotaxic frame. The nerves innervating $\mathrm{C} 2-\mathrm{C} 4 \mathrm{BCCM}, \mathrm{C} 2$ and $\mathrm{C} 3$ splenius, and trapezius muscles were mounted on stimulating electrodes. To eliminate movement of the spinal cord in response to peripheral nerve stimulation, the animals were paralyzed with gallamine triethiodide $(2.5-5 \mathrm{mg} / \mathrm{kg} / \mathrm{h}$, i.v.; Sigma) and ventilated with a respirator. Respiratory-related movements were minimized by unilateral or bilateral pnemothoraces.

\section{Injections of Neurobiotin}

We stained PCIs with multiple iontophoretic injections of Neurobiotin into the extracellular space of the ventral horn as described previously (Fenrich et al., 2007). Briefly, Neurobiotin was injected twice in each electrode track. The first injection was $200 \mu \mathrm{m}$ dorsal of the BCCM motoneuron pool, and the second was $800 \mu \mathrm{m}$ dorsal of the BCCM motoneuron pool (supplemental Fig. 1, available at www.jneurosci.org as supplemental material). Each injection was made using $5 \mu \mathrm{A}$ positive pulses with a $10 \mathrm{~s}$ on/off duty cycle for a total of $90 \mathrm{~s}$. The first electrode track used to inject Neurobiotin was aligned with the rostrocaudal position of the most caudal stimulating electrode (see below). For most of the experiments, the electrode tracks used to inject Neurobiotin were $1 \mathrm{~mm}$ apart in the rostrocaudal axis and were located at the same rostrocaudal level as the lesion, as well as regions rostral and caudal to the lesion (compare Fig. 1A). In two experiments, the electrode tracks used to inject Neurobiotin were $250 \mu \mathrm{m}$ apart in the rostrocaudal axis and were restricted to a $1 \mathrm{~mm}$ zone within the rostrocaudal confines of the lesion. These injection parameters yielded $\sim 10-20$ well stained neurons, per injection, within a sphere of $\sim 700 \mu \mathrm{m}$ in diameter. Not all of these neurons were PCIs. However, only PCIs were examined in this study. The methods used to identify PCIs are described below. After completion of the electrophysiological recordings, the cats were perfused, and the spinal cords were collected as described previously (Fenrich et al., 2007).

\section{Electrophysiology}

General methods. In each animal, we conducted one of two types of electrophysiological experiments. The first type focused on extracellular recordings of antidromically identified PCIs and are called "antidromic" experiments. The second type focused on intracellular recordings of PSPs evoked by stimulation of PCIs and are called "orthodromic" experiments. For all electrophysiological experiments, the nerves innervating the dorsal neck muscles BCCM, splenius, and trapezius were isolated and mounted on stimulating electrodes. PCIs were activated orthodromically or antidromically via electrical stimuli delivered by glass insulated tungsten electrodes (FHC). Four stimulating electrodes were spaced $2.5 \mathrm{~mm}$ apart along the rostrocaudal axis in the antidromic experiments, and six stimulating electrodes were spaced $1 \mathrm{~mm}$ apart along the rostrocaudal axis in the orthodromic experiments. The tungsten electrodes were lowered into the ventral horn beside the lesion site, and recordings of antidromic field potentials evoked by stimulation of the nerves supplying dorsal neck muscles were used to guide the placement of each stimulating electrode to regions in the ventral horn that contain the somata and axon collaterals of PCIs (Bolton et al., 1991; Sugiuchi et al., 1992, 1995). The position of the stimulating electrodes was verified histologically. To ensure that the electrophysiological, anatomical, and immunohistochemical data were in register, the rostrocaudal location of the most caudal stimulating electrode was used as a reference for positioning all of the electrophysiological and Neurobiotin electrode tracks. The shrinkage of the tissue caused by histological processing was taken into account for all alignment procedures (see below, Microscopy and image analysis). We used a Datawave DT 300 (Datawave Technologies) to collect the electrophysiological recordings and SciWorks software (version 4.1; Datawave Technologies) to display and analyze the electrophysiological recordings.

Antidromic experiments. We recorded antidromically activated PCIs using glass micropipettes (tip diameter, 1.3-2.0 $\mu \mathrm{m}$ ) filled with $2.0 \mathrm{M}$ $\mathrm{KCl}, \mathrm{pH}$ 8.0. We searched for antidromically activated PCIs using a precise grid of electrode tracks. Briefly, for each track, the recording electrode was lowered at a $6^{\circ}$ angle pointing medially to $\sim 2000 \mu \mathrm{m}$ dorsal of the motoneuron pools (based on the depth of the motoneuron field potentials recorded in the previous track or the first Neurobiotin injection). From this depth, the recording electrode was lowered in steps of 20 $\mu \mathrm{m}$. At every step along the track, we recorded the responses to sequential stimuli delivered to the tungsten electrodes. These stimuli consisted of negative, constant current pulses, $200 \mu$ s long (DS3 isolated constant current stimulator; Digitimer). The search for antidromically activated PCIs continued to a depth of $\sim 4000 \mu \mathrm{m}$ or until antidromic field potentials were observed after stimulation of the nerves supplying BBCM. Each subsequent track was $150 \mu \mathrm{m}$ rostral from the previous track and $150 \mu \mathrm{m}$ medial or lateral to optimize the magnitude of the BCCM field potentials. Antidromically activated PCIs were identified based on the following criteria: (1) a consistent response to every stimulus pulse; (2) a constant latency; (3) all or nothing at stimulus strengths near threshold; and (4) the peak-to-peak amplitude of the antidromic response was greater than twice the amplitude of the baseline noise. Based on previous studies (Shinoda et al., 1976), we chose a maximum stimulus of $95 \mu \mathrm{A}$ to mini- 
mize current spread and exclude the possibility of activating contralateral axons. Stimulus thresholds for antidromic activation of PCIs ranged from 4.5 to $92 \mu \mathrm{A}$.

Orthodromic experiments. Intracellular recordings were obtained using glass micropipettes (tip diameters, $1.3-1.8 \mu \mathrm{m}$ ) filled with $2.0 \mathrm{M} \mathrm{KCl}, \mathrm{pH}$ 8.0. We recorded from three types of neurons: (1) antidromically identified motoneurons; (2) other motoneurons; and (3) interneurons. Antidromically identified motoneurons had antidromic action potentials evoked by stimulation of nerves supplying dorsal neck muscles. Other motoneurons were located deep within the ventral horn and had action potentials with the same size and shape of typical action potentials of identified cervical motoneurons, but no antidromic action potentials were evoked by stimulation of nerves supplying dorsal neck muscles. Interneurons were located dorsal to the motoneurons, had narrower action potentials than typical cervical motoneurons, and were not antidromically activated by stimulation of nerves supplying dorsal neck muscles. We tested for PSP responses from each of the stimulating electrodes for all impaled neurons that had a stable membrane potential of at least $-35 \mathrm{mV}$. Based on previous studies (Stoney et al., 1968), we chose a maximum stimulus of $60 \mu \mathrm{A}$ to minimize current spread and exclude the possibility of activating axons rostral or caudal of the lesion zone. Stimulus thresholds to evoke PSPs ranged from 10 to $60 \mu \mathrm{A}$.

\section{Histology}

All spinal cords were processed for immunohistochemical evaluation as described previously (Fenrich et al., 2007). Briefly, Neurobiotin was visualized with streptavidin conjugated to Alexa-488 (1:100; Invitrogen). Primary antibodies raised against the following antigens were used: MAP2a/b (1:10,000; mAB AP-20; Millipore Bioscience Research Reagents), CSPG (1:250; mAB CS-56; Sigma), and synaptophysin (1:1000; polyclonal anti-synaptophysin-2; Invitrogen). Secondary antibodies were conjugated with one of the following fluorochromes: 7-amino-4methylcoumarin (AMCA), Cy3, and Cy5 (Jackson ImmunoResearch).

\section{Microscopy and image analysis}

Images were captured using an Olympus BX60 fluorescent microscope equipped with a computer-driven stage-controller and a monochrome CCD camera (Retiga EXi; QImaging). Tiled images were acquired, surveys (see below) were made using a $20 \times[0.5$ numerical aperture (NA)] objective, and reconstructions (see below) were made using $40 \times(0.75$ $\mathrm{NA})$ and $60 \times(0.95 \mathrm{NA})$ objectives. The distribution of Neurobiotin, CSPG, and MAP2a/b labeling on each tissue section was determined from tiled images that captured the entire tissue section and was analyzed using Image-Pro Plus (version 6.2; Media Cybernetics).

Evaluation of the extent of the lesion using MAP2a/b staining of dendrites at the midline. We used the absence of MAP2a/b-labeled dendrites at the midline as a marker for the extent and location of the midline lesions. The steps for determining the distribution of MAP2a/b along the midline as well as the consistency and validity of this approach are described in Results. We analyzed all serial sections that included tissue from the ventral tip of the ventral horn to the dorsal tip of the dorsal horn. If there were MAP2a/b-positive dendrites that crossed the midline in any of the serial sections, these regions were considered "unlesioned," and any Neurobiotin-labeled axons that crossed these zones were defined as uninjured, whereas any region without any MAP2a/b-positive dendrites throughout the entire dorsoventral extent of the spinal cord was considered "lesioned." The position of the lesion was aligned with the Neurobiotin injections, and the length of the lesion was corrected for tissue shrinkage according to the spacing of the Neurobiotin injections. These lesion parameters were used for all subsequent anatomical, immunohistochemical, and electrophysiological analyses. All experiments described here had lesions that transected the midline and penetrated ventrally to a minimum depth that corresponded to the ventral tips of the adjacent ventral horns.

In one 56-72 d experiment, three isolated MAP2a/b-labeled dendrites were found at different rostrocaudal locations and projected a short way across the midline. Since there were no other indicators that the lesion was incomplete, we attributed the presence of these dendrites to dendritic regeneration, and they were excluded in the evaluation of the lesion site parameters. The exclusion of these dendrites did not affect our general findings or our conclusions.

Surveys and reconstructions of Neurobiotin-labeled processes. We examined the processes labeled with Neurobiotin in two ways. Only processes that were well stained with Neurobiotin were used for both types of analyses. The first analysis was based on computer-based drawings of Neurobiotin-labeled processes located in the ventromedial funiculi along the midline. However, in the contralateral spinal cord, PCI axons project rostrally and/or caudally. For the surveys, we were interested in where processes cross the midline. Therefore, to avoid contaminating the surveys with the rostral and caudal projections of PCIs, only processes with predominantly mediolateral trajectories were included in the surveys. Each tissue section was examined independently, and no attempt was made to reconnect processes that were cut by histological sections. We refer to this method as a "survey." In contrast, reconstructions of Neurobiotin-labeled processes linked the processes cut by histological sectioning as they were followed from section to section. Thus, we could trace the three-dimensional trajectory taken by the process from the midline to the soma from which it originated. Both types of analyses were made using Neurolucida neuron tracing software (version 5.0; MicroBrightField). All surveys and reconstructions were corrected for tissue shrinkage as described above.

Surveys of Neurobiotin-stained processes at or near the midline were based on multiple tissue sections and were used to determine the rostrocaudal location of all Neurobiotin-stained processes that crossed the midline. All tissue sections with Neurobiotin-stained processes that crossed the midline were examined in each experiment (range, 8-26 sections per animal; average, 15 sections across all animals). The drawing of each survey began by tracing Neurobiotin-stained processes along and near the midline in a section, followed by tracing the outline of the tissue section. A composite of the individually traced tissue sections was compiled by overlaying the tracings and were aligned according to the outlines of the tissue section. These surveys show the distribution of Neurobiotin-stained processes at or near the midline for the entire dorsoventral extent of the spinal cord.

Neurobiotin injections stained a variety of neurons, including PCIs. Therefore, all reconstructions began by following Neurobiotin-labeled axons that projected toward the midline. Only those axons that could be traced back to a soma in the adjacent gray matter were considered to arise from PCIs, and only reconstructions of PCIs were included in this study. All reconstructions began with a thorough search of the lesion site for processes that crossed the midline or approached the midline from the injection sites. For $0 \mathrm{~d}$ experiments, reconstructions began at the cut axon terminals near the lesion (i.e., retraction bulbs). For $7 \mathrm{~d}$ and 56-72 $\mathrm{d}$ experiments, all reconstructions began at a growth cone in the lesion site or at a process that crossed the midline within the lesion. Reconstructions thus provided a detailed morphological representation of PCI axons and their somata and the locations of these axons and somata and immunohistochemical markers.

Quantitative analysis of CSPG labeling. We measured the average intensity of CSPG labeling within regions of interest (ROIs) at the lesion site and lateral white matter for all experiments. All images of CSPG labeling were collected using the same acquisition settings and exposure times. For this analysis, we used a secondary antibody conjugated to AMCA to visualize the distribution of CSGP and Vectashield as a mounting medium. However, Vectashield caused a faint blue autofluorescence. To reduce the incidence of false-positive CSPG labeling, the magnitude of the blue autofluorescence caused by Vectashield (as measured in regions immediately adjacent to each tissue section) was subtracted from the blue fluorescence measured in the ROIs. There was no difference between the relative intensity of CSPG labeling using this method compared with similar measures based on tissue that was mounted with a mounting medium that does not autofluoresce blue (Citifluor) or after bleaching the blue autofluorescence of the Vectashield (data not shown).

In the $0 \mathrm{~d}$ experiments, all tissue sections that had retraction bulbs were analyzed for CSPG intensity. In the $7 \mathrm{~d}$ experiments, all tissue sections that had growth cones near the lesion were analyzed for CSPG intensity. In the 56-72 d experiments, all tissue sections that had growth cones near the lesion or axons crossing the lesion site were analyzed for 
CSPG intensity. The rostrocaudal locations of the ROIs were determined by the rostrocaudal location of the lesion, and the width of the ROIs was set at $390 \mu \mathrm{m}$.

Within ROIs, there were often regions without spinal tissue (i.e., tissue voids) or regions with autofluorescent dust particles (see Fig. 5). Tissue voids were easily detected by the absence of fluorescence regardless of which fluorochrome was examined. Tissue voids were traced using the images of Neurobiotin-labeled processes. Dust particles are defined as intensely fluorescent regions that are not in the same focal plane as the tissue (i.e., are between either the tissue and the slide or the tissue and the coverslip). Both tissue voids and dust particles were excluded from the regions used for measurements of CSPG intensity.

Quantitative analysis of synaptophysin labeling. To determine the distribution of synaptophysin within the boutons of uninjured and regenerated PCI axon collaterals, images of the Neurobiotin and synaptophysin labeling were acquired at multiple focal planes with a $60 \times(0.95 \mathrm{NA})$ objective. The focal plane of each set of images was controlled by a computerdriven stage-controller, and each focal plane was separated by $1 \mu \mathrm{m}$. Neurobiotin-stained swellings were defined as boutons if their diameter was at least twice that of the parent shaft (Grande et al., 2005). Synaptophysin-positive boutons were those with punctate synaptophysin labeling that was colocalized to the bouton when viewed in the $x$-and $y$-axes and at least two adjacent focal planes when viewed in the $z$-axis. Synaptophysin-negative boutons were those with no synaptophysin labeling. These criteria assume that the fluorescence generated by synaptophysin in synapses adjacent to Neurobiotin-stained boutons does not "spread" into the volume of tissue occupied by the bouton. To test this assumption, we examined the registration of objects viewed with the same filter sets used to image Neurobiotin and synaptophysin, using 4 - $\mu \mathrm{m}$-diameter TetraSpecs fluorescent microspheres (Invitrogen). The red and green fluorescence emitted by the TetraSpecs were colocalized to within 1 pixel $(0.2 \times 0.2 \mu \mathrm{m})$ in the $x$ - and $y$-axes and to within one focal plane in the $z$-axes (data not shown). Thus, our assumption was justified.

\section{Statistics}

Statistical significance was determined using the Kruskal-Wallis one-way ANOVA, the Mann-Whitney $U$ test, or the Kolmogorov-Smirnov test (Systat; SPSS). The specific tests used for each evaluation are described in the figure legends.

\section{Results}

\section{PCI axons are cut by a midsagittal spinal lesion}

The axons of PCIs were cut at the point where they cross the midline with a midsagittal cut of the spinal cord at C3 (Fig. 1A). This study had three postaxotomy intervals. Animals who had received a midline transection several hours before fixation were called 0 d experiments, and animals that received a midline transection $7 \mathrm{~d}$ or $56-72 \mathrm{~d}$ before fixation were called $7 \mathrm{~d}$ and $56-72$ $\mathrm{d}$ experiments, respectively. The approximate locations of the midline lesions were easily identified on serial horizontal sections that contained gray matter. These sections had a longitudinal hole running along the midline in $0 \mathrm{~d}$ experiments, dense CSPG labeling along the midline in the $7 \mathrm{~d}$ and $56-72 \mathrm{~d}$ experiments (see below), and a consistent lack of MAP2a/b-labeled dendrites at the midline in all experiments (see below). Since the absence of MAP2a/b at the midline is the only consistent feature of the lesion site for all postaxotomy intervals, we used the absence of MAP2a/ b-labeled dendrites at the midline as a marker for the lesion. However, since studies of this sort are susceptible to false-positive conclusions of regeneration (Steward et al., 2003), we determined whether the absence of MAP $2 \mathrm{a} / \mathrm{b}$ dendrites at the midline is an accurate indicator of the extent and position of the spinal lesions regardless of the postinjury interval.

To test this, we first examined whether the midsagittal transection protocol cuts PCI axons at the midline in the $0 \mathrm{~d}$ and $7 \mathrm{~d}$ experiments. During these experiments, Neurobiotin was injected into the extracellular space of the ventral horn at the same rostrocaudal level of the lesion. We constructed surveys (see Materials and Methods) of the Neurobiotin-stained processes at the midline from these experiments to examine the distribution of processes along the midline (Fig. $1 B, C)$. At both $0 \mathrm{~d}(n=3)$ and $7 \mathrm{~d}$ $(n=3)$ after injury, there were no Neurobiotin-stained processes that crossed the midline within a zone that was equivalent to the intended length of the transection ( 3.5 or $4 \mathrm{~mm}$ ). PCI axons and dendrites from a variety of cells cross the spinal midline from the ventral commissure to the anterior median fissure (supplemental Fig. 1, available at www.jneurosci.org as supplemental material). Since dendrites cross the midline throughout the entire dorsal ventral extent through which PCI axons cross the midline, we determined whether regions along the midline without MAP2a/ b-labeled dendrites match the regions where Neurobiotinstained processes are also absent. The lesioned zones were defined by those regions without any midline MAP2a/b labeling as observed in all of the serial sections. We first determined the rostral edge of the lesion. For each section, the MAP2a/b-labeled dendrite that crossed the midline just rostral to the zone without MAP2a/b dendrites was identified. The rostrocaudal position of this dendrite was measured relative to the rostrocaudal location of a predetermined Neurobiotin injection site (Fig. 1 E, F). These steps were repeated for all serial sections that included tissue from the ventral tip of the ventral horn to the dorsal tip of the dorsal horn. The position of the most caudal dendrite based on all these sections was defined as the rostral edge of the lesion. This process was repeated to determine the caudal edge of the lesion, except the most rostral dendrite at the edge of the lesion was used to define the caudal edge. This method for defining the extent of the lesion yielded the smallest region without MAP2a/b-labeled dendrites along the midline (Fig. $1 G$ ).

We compared the distribution of Neurobiotin-stained axons at the midline with the distribution of MAP2a/b-labeled dendrites at the midline for all $0 \mathrm{~d}$ and $7 \mathrm{~d}$ experiments. This analysis consistently indicated that midline regions without MAP2a/b labeling (Fig. 1B,C, red lines overlaid on surveys) do not have spared axons and midline regions with MAP2a/b labeling have intact axons. These data indicate that the midline regions without $\mathrm{MAP} 2 \mathrm{a} / \mathrm{b}$ dendrites provide an accurate measure of the rostrocaudal extent of the transection for the $0 \mathrm{~d}$ and $7 \mathrm{~d}$ experiments.

For the 56-72 d experiments, we collected surveys of Neurobiotinstained processes located at the midline (Fig. 1D) $(n=8)$ and determined the rostral-to-caudal region lacking MAP2a/bstained dendrites as described for the $0 \mathrm{~d}$ and $7 \mathrm{~d}$ experiments. To determine whether the absence of MAP $2 \mathrm{a} / \mathrm{b}$ dendrites at the midline is a valid indicator of the location and extent of where PCI axons were cut along the midline at later postinjury times, we compared the lengths of the regions without MAP $2 \mathrm{a} / \mathrm{b}$ from the different postaxotomy times. There were no significant differences between the average rostral-to-caudal length of these regions at 0,7 , and 56-72 d after injury ( $p>0.05$, Kruskal-Wallis one-way ANOVA; data not shown). Together with the other observations, the regions lacking MAP2a/b-immunoreactive dendrites at the midline are an accurate indicator of both the extent and location of where commissural axons were cut from 0 to $72 \mathrm{~d}$ after lesion.

\section{PCI axons regenerate through the lesion site}

The large numbers of Neurobiotin-stained processes that crossed the midline at the site of the lesion (Fig. 1D) suggest that transected axons from PCIs have regenerated through the injury site by 56-72 d after lesion. However, these observations do not provide morphological information regarding the path followed by indi- 
A

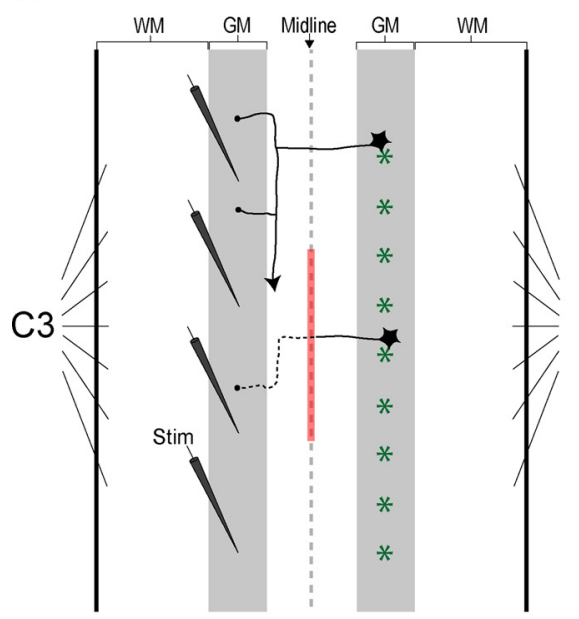

B

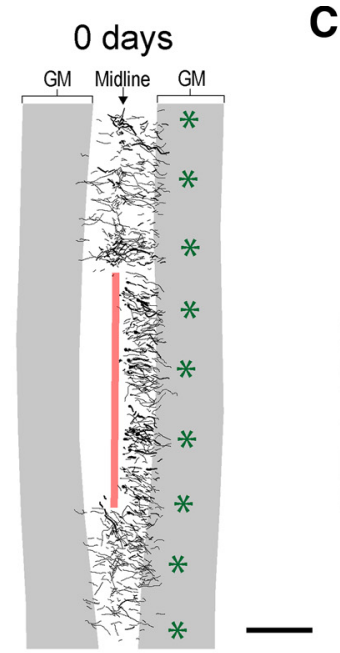

C

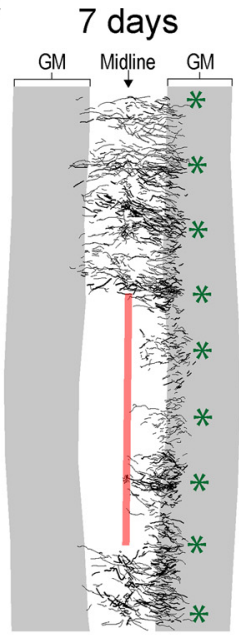

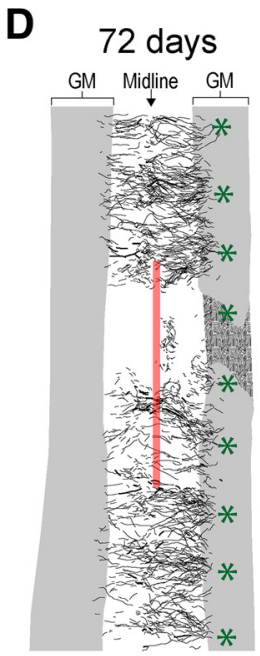

G

E
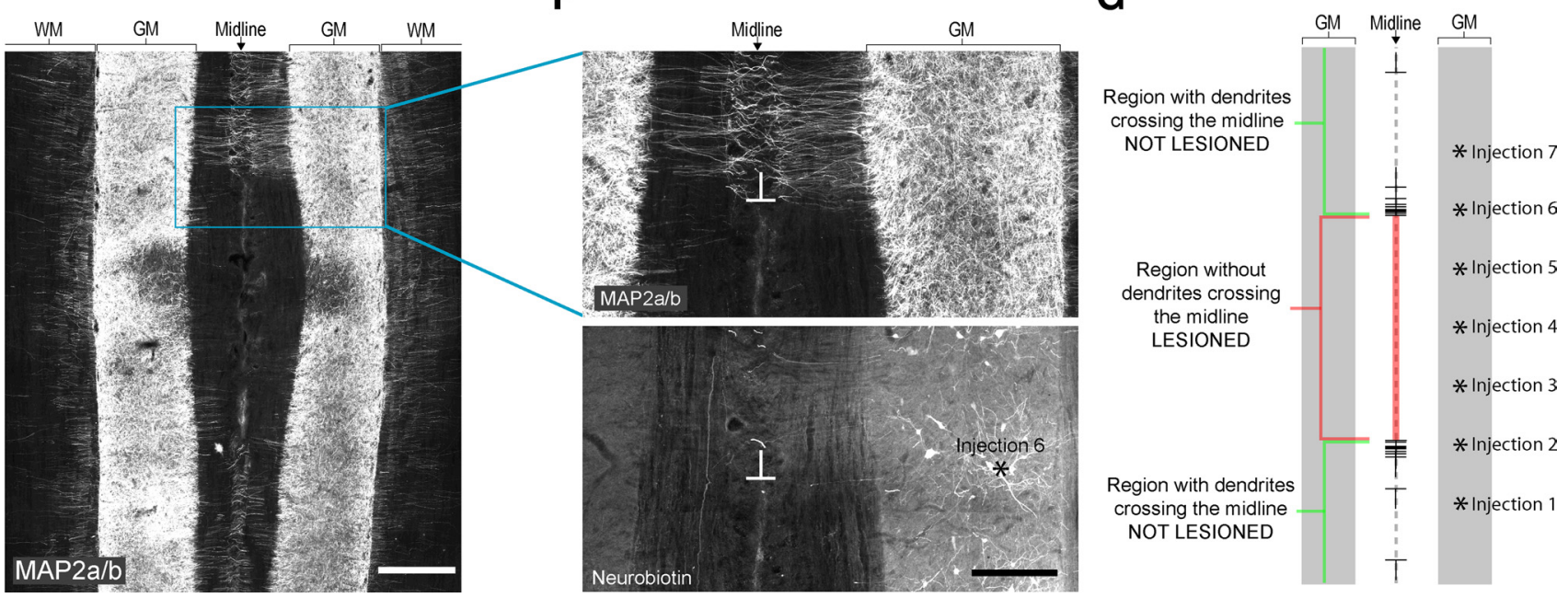

Figure 1. Morphological characteristics of midsagittal lesions observed at different postinjury intervals. $A$, Schematic of the $C 3$ spinal cord as viewed in the horizontal plane, showing a midline lesion (red line) and PCl axotomy. Green asterisks indicate the location of Neurobiotin injections. Stim, Stimulation. $\boldsymbol{B}-\boldsymbol{D}$, Representative surveys of Neurobiotin-stained processes at the midline for $0 \mathrm{~d}(\boldsymbol{B}), 7 \mathrm{~d}(\boldsymbol{C})$, and $72 \mathrm{~d}(\boldsymbol{D})$ experiments. Neurobiotin processes are shown in black. Red lines indicate regions at the midline where crossing axons were transected as determined by the distribution of MAP2a/b-labeled dendrites near the midline. The hatched area in the right gray matter of $\boldsymbol{D}$ indicates damaged gray matter (see Results, Regenerated PCl axons conduct action potentials). Green asterisks indicate the location of Neurobiotin injections. Scale bar, $1 \mathrm{~mm}$. $\boldsymbol{E}$, Tiled images of MAP2a/b labeling in the white matter, gray matter, and along the midline (including the lesion site) of a representative section from a $0 \mathrm{~d}$ experiment. Scale bar, $1 \mathrm{~mm}$. $\boldsymbol{F}$, Enlarged view of the rostral edge of the lesion shown in $\boldsymbol{E}$. The top panel shows MAP2a/b labeling, and the bottom panel shows a Neurobiotin injection site in the corresponding region. The horizontal edge of the $\perp$ symbol indicates the rostral boundary that separates a rostral region that contained midline MAP2a/bimmunoreactive dendrites from a caudal region where MAP2a/b-immunoreactive dendrites did not cross the midline on this section. Scale bar, $0.5 \mathrm{~mm}$. G, Schematic description of the methods used to determine the extent and location of the lesion based on analysis of MAP2a/b labeling at the midline of multiple serial tissue sections. The horizontal edge of the $\perp$ and $T$ symbols indicate the rostrocaudal boundaries, respectively, that separated midline regions with and without MAP2a/b-immunoreactive dendrites. Each symbol shows the boundary for an individual tissue section. The most caudal $\perp$ across from injection 6 defines the rostral edge of the lesion, and the most rostral $T$ across from injection 2 defines the caudal edge of the lesion. WM, White matter; GM, Gray matter.

vidual axons, and therefore we could not differentiate between regenerated axons and collaterals that may have emerged from uninjured axons near the lesion. Therefore, we traced the path followed by axons that projected toward or through the midline lesion site.

At $0 \mathrm{~d}$ after injury, although the surveys showed that there were many Neurobiotin-stained processes that projected toward the lesioned midline, we had no evidence of regeneration across the lesion site (Fig. 1 B). To confirm that the Neurobiotin-stained processes that approached the midline were the cut axons of PCIs, we performed reconstructions of these Neurobiotin-stained processes (Fig. 2A). All of these processes had morphological features that were consistent with axons (e.g., did not taper en route from the soma). None of these axons crossed the midline lesion ( $n=0$ of 39), all had bulbous terminals (Fig. $2 A$ ), and all were traced to somata in the adjacent gray matter. A detailed examination of the other processes emerging from the somata revealed no other axons (i.e., all of the processes were dendrites as defined by rapid tapering and acute angle branching) and were therefore considered axotomized PCIs. We also performed reconstructions of Neurobiotin-stained processes that approached the midline for all $7 \mathrm{~d}$ experiments. All of the processes had morphological features typical of axons and were traced to somata in the adjacent gray mater. These somata had no other axons and were therefore considered axotomized PCIs. One of the PCI axons in the $7 \mathrm{~d}$ experiments crossed the midline lesion $(n=1$ of 27 ; reconstruction not shown). The main axon of this PCI was traced to an abrupt bulbous termination in the contralateral ventral medial funiculus and did not enter the gray matter. All other reconstructed axons from $7 \mathrm{~d}$ experiments terminated before or 
within the lesion site (Fig. $2 B$ ). The terminals of these PCI axons varied in complexity; from a simple swelling at the end of the axon, to structures with many branches, tortuousities, and varicosities. These terminals were called growth cones.

At 56-72 d after injury, there were many Neurobiotin-stained processes that crossed the lesion site according to our surveys of Neurobiotin-stained processes (Fig. 1D). Thirty-three of these processes within the lesion site were followed to somata in the adjacent gray matter, and all had morphological features typical of axons (Fig. 2C). Although several of these axons had fine collaterals that branched off of the main shaft (see below), the main shaft did not bifurcate en route to the soma as would be expected if the regenerated axons were collateral branches of a main axon that projected elsewhere. A detailed examination of the other processes emerging from the somata revealed no other axons. Thus, we concluded that the processes at the lesioned midline were extensions of the cut PCI axons that had regenerated subsequent to the injury and were not collaterals from uninjured axons.

Of the 33 axons described above, 26 traversed the lesion and entered the contralateral ventromedial funiculus (Fig. 2C) (data collected from four animals; $n=7,3,15$, and 1 , respectively). Ten of these axons crossed the midline lesion in the caudal third of the lesion, nine crossed in the middle third of the lesion, and the remaining seven crossed in the rostral third of the lesion (supplemental Fig. 2, available at www.jneurosci.org as supplemental material). Thus, the location at which regenerating axons crossed the lesioned midline appeared to be independent of their position relative to the lesion. This wide distribution along the rostrocaudal length of the lesion was also a feature of the 15 axons that were examined in one experiment. In the contralateral cord, most of these axons projected rostrally and/or caudally with a gradual ventral progression and formed collaterals in the contralateral ventral horn. These morphological features are typical for cervical PCI axons (see below and Sugiuchi et al., 1992, 1995). It should be noted that the ratio of 26 to 33 is likely an overestimate of the proportion of PCI axons that regenerate into the contralateral spinal cord. All of the reconstructions began at or near the midline, and we therefore excluded axons that may have retracted to the soma or axons from PCIs that died because of the injury. The reconstructed PCI axons that did not cross the midline lesion ended in growth cones near the midline (Fig. 2C). Axons that terminated in growth cones of the 56-72 d experiments tended to have fewer branches and swellings and were longer with more tortuous trajectories than axons with growth cones of $7 \mathrm{~d}$ exper-
B

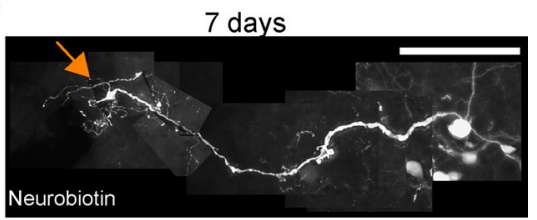

Reconstruction

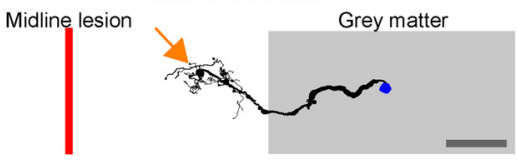

72 days

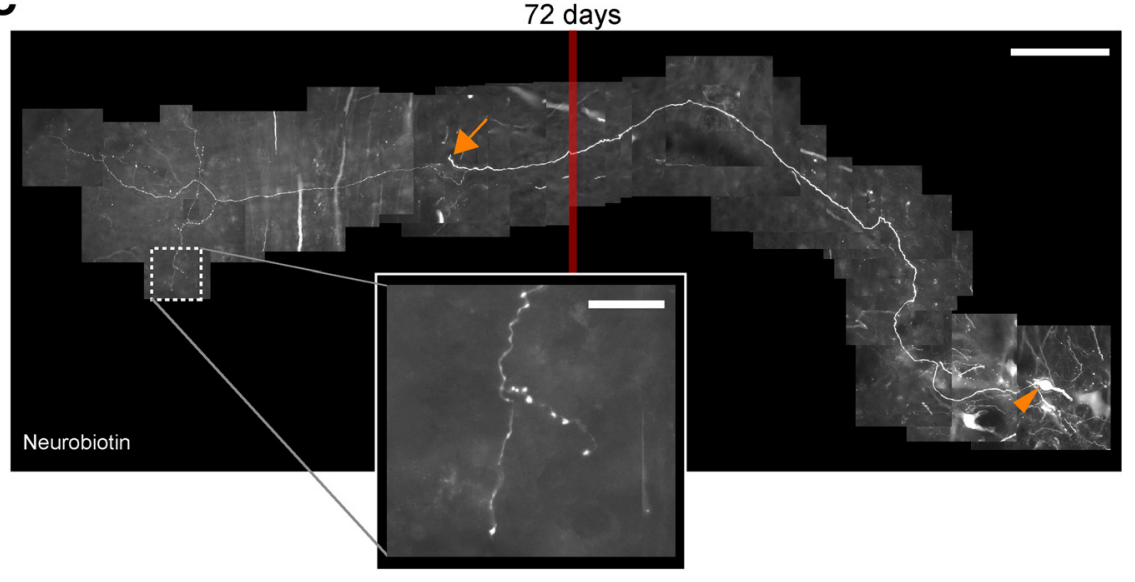

Reconstructions

Grey matter Midline lesion Grey matter

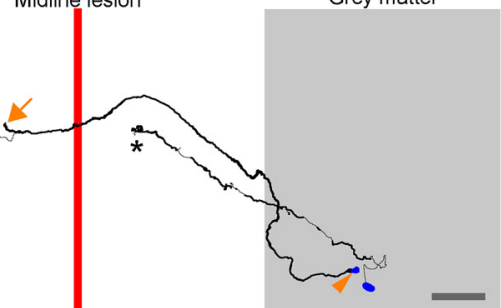

Figure 2. $\mathrm{PCl}$ regenerate through the lesion site after axotomy. $\boldsymbol{A}-\boldsymbol{C}$, Top, Mosaic images of axotomized PCls stained with Neurobiotin. Bottom, Reconstructions of the PCls shown in the top panels (black, axons; blue, somata). $\boldsymbol{A}, \mathrm{PCl}$ axon $0 \mathrm{~d}$ after the location of the bulbous terminal relative to midline lesion. Scale bars, $200 \mu \mathrm{m}$. B, PCl axon $7 \mathrm{~d}$ after axotomy. The mosaic ( $n=$ 8 images from 4 serial sections) shows a complex growth cone (arrow). The reconstruction indicates the location of growth cone hows a representative $\mathrm{PCl}$ axon that crosses the midline lesion (red line) and forms collaterals (left and inset). The arrowhead $\mathrm{PCl}$ axon that did not regenerate across the midline lesion and terminated in a growth cone (asterisk). Scale bars: mosaic and reconstruction, $200 \mu \mathrm{m}$; inset, $25 \mu \mathrm{m}$.

iments. These axons may account, in part, for the high density of Neurobiotin processes near the midline lesion in the $56-72 \mathrm{~d}$ experiments (compare Fig. $1 D$ ).

As seen in the $0 \mathrm{~d}$ and $7 \mathrm{~d}$ experiments, in the 56-72 $\mathrm{d}$ experiments, axons crossing the lesion site were not detected in certain parts of the lesions (supplemental Fig. 2, available at www.jneurosci. org as supplemental material). Lesioned zones without regenerated PCI axons in the 56-72 d experiments were always immediately adjacent to regions that had very little electrophysiological activity (i.e., few or no spontaneous or injury discharges; see below, Regenerated PCI axons conduct action potentials), very few Neurobiotin-labeled somata, no MAP2a/b labeling, and strong CSPG labeling (supplemental Fig. 3, available at www. 
A

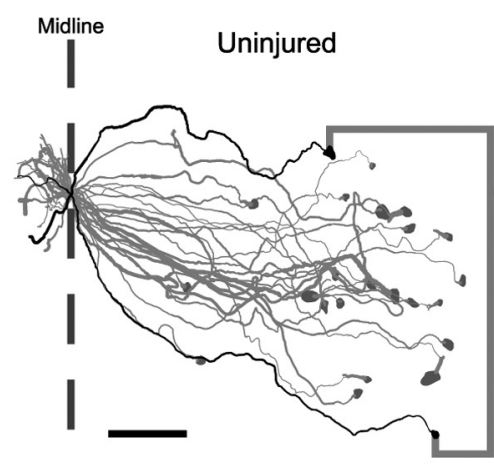

Midline lesion

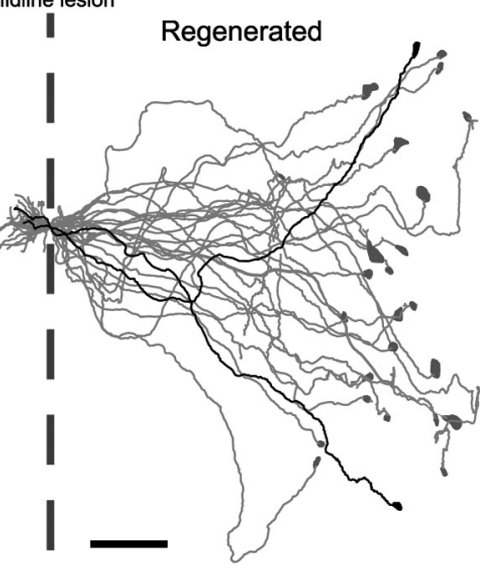

B

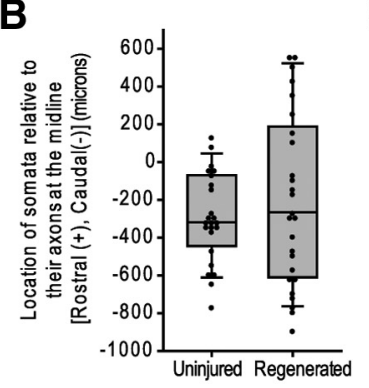

C

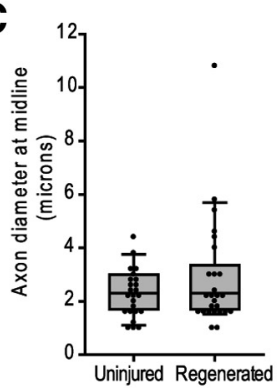

D

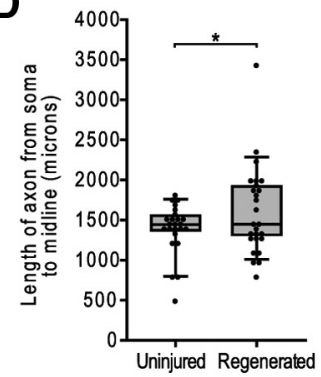

Figure 3. Regenerated $\mathrm{PCl}$ axons are morphologically similar to uninjured $\mathrm{PCl}$ axons. $\boldsymbol{A}$, Reconstructions of intact (left; $n=24$; 13 were derived from one animal, and the remaining were based on data collected in a second animal) and regenerated (right; $n=$ 26) $\mathrm{PCl}$ axons that crossed the midline (reconstructions continue in contralateral spinal cord but have been truncated for clarity). The reconstructions are shown in the horizontal plane and are aligned rostrocaudally to the point at which each axon crossed the midline. Reconstructions in black show the $\mathrm{PCl}$ axons with the most rostral and caudal somata relative to the location at which their axons crossed the midline. The parameters used for calculating the transition zones of the 'antidromic' experiments (see Results, Regenerated PCl axons conduct action potentials) are shown on the right of the uninjured reconstructions. Scale bars, $1 \mathrm{~mm}$. $\boldsymbol{B}, \boldsymbol{C}$, Quantification of the morphological parameters of intact and regenerated $\mathrm{PCl}$ axons. $\boldsymbol{B}$, Box plot showing the rostral-to-caudal difference between the locations of $\mathrm{PCl}$ somata relative to the location at which their axons crossed the midline. C, Box plot showing the diameter of $\mathrm{PCl}$ axons measured at the midline. $\boldsymbol{D}$, Box plot showing the length of the axon between the $\mathrm{PCl}$ somata and the location at which their axons crossed the midline $\left({ }^{*} p<0.05\right.$, Kolmogorov-Smirnov test).

jneurosci.org as supplemental material). We presume that these regions were severely compromised by the midline lesion (i.e., vascular or compressive damage) (Fig. $1 D$, hatched area; supplemental Fig. 3, starred arrow, available at www.jneurosci.org as supplemental material) and that few neurons survived to regenerate from these regions. Similar regions were observed in the $0 \mathrm{~d}$ and $7 \mathrm{~d}$ experiments and were always associated with a lack of antidromically activated PCIs, similar Neurobiotin and MAP2a/b staining characteristics, and little electrophysiological activity (data not shown).

To compare the morphologies of uninjured and regenerated PCI axons, we reconstructed uninjured PCI axons $(n=23)$ from $0 \mathrm{~d}$ experiments. These axons were located rostral and caudal of the lesion sites. As described above, the reconstructions started at the midline and followed the axon to its soma of origin (Fig. 3A). We also traced each of these axons for several hundred micrometers en route to their terminations in the contralateral spinal cord. Uninjured PCI axons typically projected dorsally and medially from the soma and crossed the midline in the ventral commissure. In the contralateral cord, the axons projected rostrally, caudally, or bifurcated and projected both rostrally and caudally (data not shown). Other studies have shown that uninjured PCI axons typically project rostrally and/or caudally for several millimeters in the contralateral ventral funiculus and send collaterals into the adjacent ventral horn throughout their length (Sugiuchi et al., 1992, 1995). We also compared the rostrocaudal location of PCI somata relative to the location at which their axons cross the midline (Fig. $3 B$ ), the axon diameter at the midline (Fig. $3 C$ ), and the length of the axon from the soma to the midline (Fig. 3D). The characteristics of regenerated $\mathrm{PCI}$ axons tended to be more variable than uninjured PCI axons. The average axon length from the soma to the point at which it crosses the midline was significantly longer for regenerated axons than intact axons $(p<0.05)$ (Fig. 3D). Also, several regenerated axons $(n=4$ of 23) formed collaterals before crossing the midline, whereas the uninjured axons had none.

These results indicate that PCI axons regenerate through the lesion site between 7 and 56-72 d after injury and that regenerated PCI axons are morphologically similar to intact PCI axons.

\section{PCI axons rarely regenerate rostral or caudal to the lesion site}

Although the data shown in the previous sections indicate that the axons of PCIs regenerate through the lesion sites, these data do not preclude the possibility that some regenerating PCI axons followed a circuitous route whereby they projected rostrally or caudally and skirted the lesion (Fig. 4A, diagram, bottom cell). Therefore, to determine whether cut PCI axons cross the midline rostral or caudal of the lesion site, we performed two $56 \mathrm{~d}$ experiments as outlined in Figure $4 A$, in which the Neurobiotin injections were restricted to gray matter adjacent to the lesion. Because of extensive scarring on the dorsal surface of the spinal, it is not possible to use anatomical markers as a means of accurately determining the rostrocaudal location of the lesion at 8-10 weeks after axotomy. Therefore, to increase the probability of restricting the Neurobiotin injections to within the rostrocaudal confines of the lesion, the lesions in these experiments were $6 \mathrm{~mm}$ long in the rostrocaudal plane. Neurobiotin injections were made $250 \mu \mathrm{m}$ apart in the rostrocaudal plane and were restricted to a $1 \mathrm{~mm}$ zone of gray matter adjacent to the lesion.

In one experiment, all of the Neurobiotin injections were well within the rostrocaudal confines of the lesion (Fig. 4B). Many Neurobiotin-stained processes were observed at the midline within the lesion site and across the lesion site contralateral of the Neurobiotin injections. One process crossed the midline $\sim 1 \mathrm{~mm}$ rostral of the lesion site. The presence of this axon rules out the possibility that the absence of axons rostral or caudal to the lesion is attributable to the inability of the axons to transport Neurobiotin for long distances. In the experiment shown in Figure $4 C$, the rostral three injections were within or very close to damaged gray matter and therefore stained very few cells. The most caudal Neurobiotin injection was immediately adjacent to the caudal edge of the lesion and was therefore in a location that contained a combination of axotomized and uninjured PCIs (compare Fig. 3A). Despite the positioning of the injections in this experiment, many 
Neurobiotin-stained processes were observed at the midline within the lesion site and across the lesion site contralateral of the Neurobiotin injections. Also in this experiment, one process crossed the midline $\sim 1 \mathrm{~mm}$ caudal of the lesion site. In both experiments, all of the Neurobiotinstained processes that crossed the midline had morphological features typical of axons and were not MAP2a/b immunoreactive, thus indicating that these processes were likely regenerated axons. The experiments shown in Figure 4 indicate that the vast majority of regenerating PCI axons grow through the lesion site rather than rostral or caudal of the lesion site.

\section{PCI axons regenerate through a CSPG-} rich environment

Given the ability of some PCI axons to grow through the lesion site after axotomy, we next determined whether the lesion site contained inhibitory molecules. We tested for the presence of CSPGs at the lesion site, since previous studies have shown that CSPGs cause growth cone stalling (Tom et al., 2004) and regeneration failure (McKeon et al., 1991). In addition, since CSPGs within the glial scar are produced by reactive astrocytes, the distribution of intense CSPG may be considered a rough estimate of the distribution of reactive astrocytes. This is consistent with Davies et al. (1999), who showed consistent overlap between CSPG and GFAP labeling after dorsal hemisections in the rat spinal cord. The distribution of CSPGs in the midline at each postinjury interval had a distinct labeling pattern. At $0 \mathrm{~d}$, there was little or no CSPG staining at the lesion site (Fig. 5A). At $7 \mathrm{~d}$ (Fig. 5B) and 56-72 d (Fig. 5C) after injury, CSPG labeling was clearly visible throughout the rostrocaudal extent of the midline lesion and extended several hundred micrometers lateral. At $7 \mathrm{~d}$ after injury, the CSPG labeling formed "tube-like" structures throughout the lesion site (Fig. 5, compare B, F). By 56-72 d after injury, CSPG labeling was more uniform and either formed a "ring-like" pattern around the lesion or filled the center of the lesion (Fig. 5, compare $C, G$ ).

The presence of CSPGs at the lesion site in the $7 \mathrm{~d}$ experiments, before regeneration, and in the 56-72 d experiments, after regeneration, suggests that PCI axons regenerate through an inhibitory environment. To confirm that CSPG was upregulated before regeneration, the average density of CSPG labeling was quantified. The amount of CSPG labeling in the lesion site was compared with the amount of CSPG labeling in the lateral white matter (excluding regions without tissue and autofluorescent dust particles; see Materials and Methods) (Fig. 5D). We found that there was significantly more CSPG at the midline lesion site than the lateral white matter in the $7 \mathrm{~d}$ and 56-72 $\mathrm{d}$ experiments $(p<0.05)$ (Fig. 5E). However, there was no difference between the density of the CSPG labeling at the lesion site and lateral white matter in $0 \mathrm{~d}$ experiments. This indicates that CSPGs are typically expressed at low levels in the intact spinal cord but are upregulated by $7 \mathrm{~d}$ after the lesion and maintained at the injury site for at least $72 \mathrm{~d}$ after a midline spinal lesion. We also examined the CSPG labeling adjacent to the growth cones of the $7 \mathrm{~d}$ experiments and the regenerated PCI axons of the 56-72 d experiments. We found that the growth cones in $7 \mathrm{~d}$ experiments were closely apposed to the CSPG labeled tube-like structures (Fig. $5 F$ ) and that all of the regenerated axons of the 56-72 d experiments crossed regions of dense CSPG labeling (Fig. $5 G$ ). These results show that midline lesions contain molecules that potently inhibit regenerating axons and that PCI growth cones are in close association with these molecules while crossing the lesion site.

\section{Regenerated PCI axons conduct action potentials}

Since the conduction of action potentials is an inherent requirement for functional regeneration, we tested the ability of regenerated PCI axons to conduct action potentials. PCIs were activated antidromically by stimulation through an array of stimulating electrodes in the contralateral spinal cord (Fig. $6 A)$. Antidromic responses of stimulated PCIs were recorded extracellularly (Fig. $6 B$ ). These electrophysiological experiments were performed in the same animals that were used for the studies of Neurobiotin-labeled processes, the distribution of MAP2a/b dendrites, and the distribution of CSPG $(0 \mathrm{~d}, n=3$ animals; $7 \mathrm{~d}$, $n=3$ animals; $56-72 \mathrm{~d}, n=3$ animals). Based on histological analysis of the lesion, the gray matter was divided into three rostrocaudal zones: gray matter that contained uninjured PCIs (uninjured zone), transition gray matter (transition zone), and gray matter that contained axotomized PCIs (lesioned zone) (Fig. $6 A)$. Recordings were made in all three zones. Transition zones were devised to reduce the possibility of recording antidromic responses from uninjured PCIs and attributing them to regenerated PCI axons. The transitions zones thus contain a mixture of PCI somata, whose axons were cut by the midline lesion and those whose axons were not cut by the lesion. The extent of the transition zones were based on the rostral-to-caudal trajectory followed by axons of uninjured PCIs en route from their somata to the midline. As shown in Figure $3 A$, the maximum caudal distance traveled by PCI axons before crossing the midline was $138 \mu \mathrm{m}$. The maximum rostral distance traveled by PCI axons before crossing the midline was $760 \mu \mathrm{m}$. To these distances, we added $100 \mu \mathrm{m}$ (50 $\mu \mathrm{m}$ rostral and $50 \mu \mathrm{m}$ caudal $)$ to account for the spread of extracellularly recorded antidromic action potentials (based on the recordings from antidromi- 
A

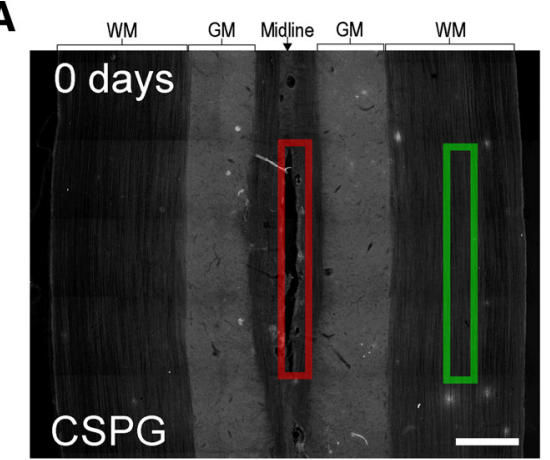

B
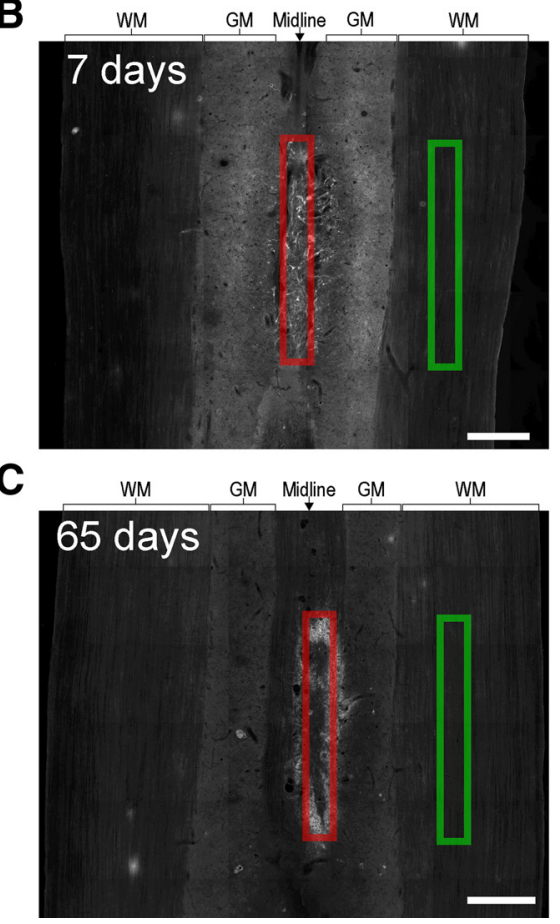

D

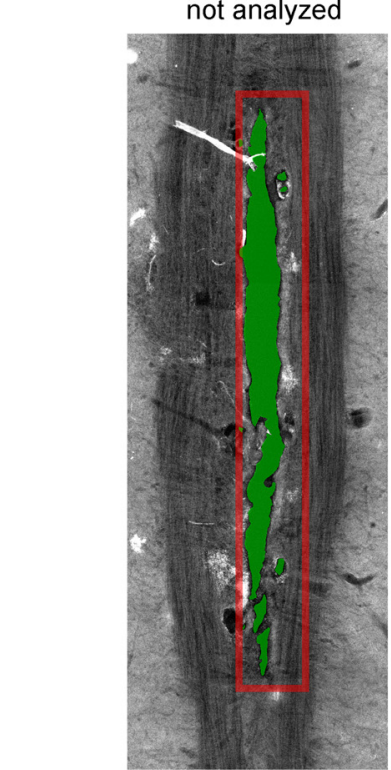

Dust particles not analyzed

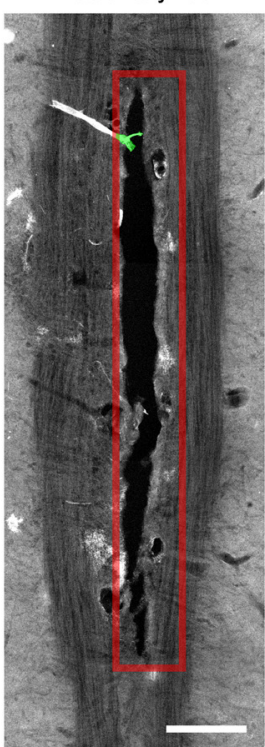

E

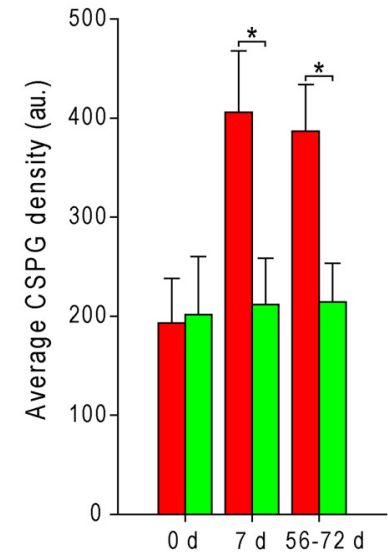

F

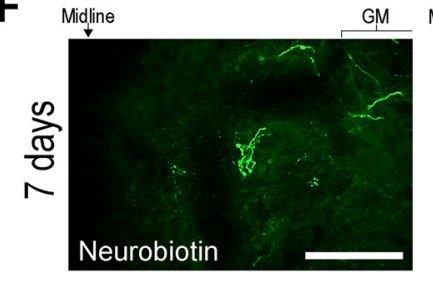

G

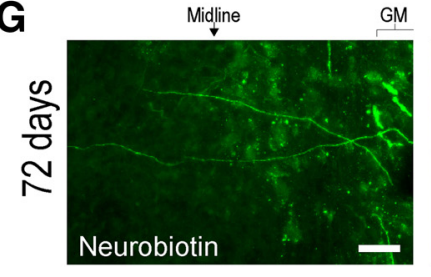

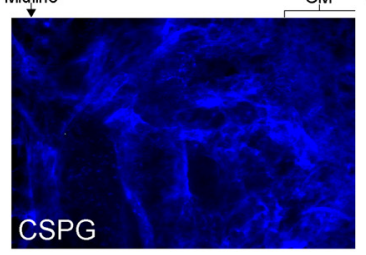

Midline

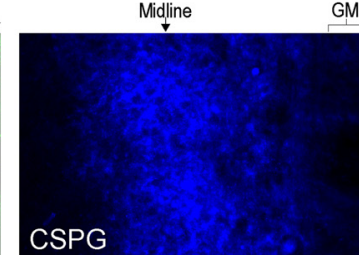

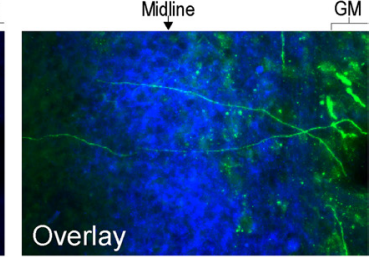

Figure 5. $\mathrm{PCl}$ axons regenerate through an environment containing CSGP immunoreactivity. $A-C$, Representative tiled images of CSPG labeling at the lesion site and surrounding areas at $0 \mathrm{~d}(\boldsymbol{A})$, $7 \mathrm{~d}(\boldsymbol{B})$, and $65 \mathrm{~d}(\boldsymbol{C})$ after lesion. Red rectangles represent the ROls for analysis of the density of CSPG labeling in the lesion site for that tissue section; green rectangles represent the ROIs for analysis of the density of (SPG labeling in the lateral white matter for that tissue section. Scale bars, $1 \mathrm{~mm}$. $\boldsymbol{D}$, Enlarged images of the lesion site shown in $\boldsymbol{A}$ (intensity increased for the purposes of illustration). Green areas show the regions without tissue (left) and regions of dust particles (right). These areas were excluded from the analysis of CSPG density. Scale bar, $0.5 \mathrm{~mm}$. $E$, Bar graphs showing the average density of CSPG labeling per animal ( $n=3$ for 0 d experiment; $n=3$ for 7 dexperiment; $n=8$ for $56-72 \mathrm{~d}$ experiment). Values represent means \pm SEM ( ${ }^{*} p<0.05$, Mann-Whitney $U$ test). $\boldsymbol{F}$, Representative example of a PCl growth cone at $7 \mathrm{~d}$ after lesion and its close apposition with (SPG labeling. Scale bar, $100 \mu \mathrm{m}$. $\mathbf{G}$, Representative example of regenerated PCl axons that cross the midline lesion at $72 \mathrm{~d}$ after lesion. The lesion was densely labeled for CSPG. Scale bar, $100 \mu \mathrm{m}$. WM, White matter; GM, gray matter.

cally activated PCIs; data not shown) (Fig. 3A). Thus, the total length of the transition zone was almost $1000 \mu \mathrm{m}$. These zones were aligned with the rostral and caudal borders of the lesions (Fig. 6A, triangular zones), as defined by the MAP2a/b labeling.

At 0 and $7 \mathrm{~d}$ after injury, multiple antidromically activated PCIs were recorded in most electrode tracks within the uninjured zones. Fewer PCIs were recorded in the transition zones, and none were recorded in the lesioned zones (Fig. 6C). At 56-72 d after injury, we also identified multiple antidromically activated PCIs recorded in most electrode tracks within the uninjured zone and, similar to the experiments at 0 to $7 \mathrm{~d}$ after injury, fewer antidromically activated PCIs were recorded in the transition zones. In contrast, by 56-72 d after injury, antidromically activated PCIs were recorded in the lesioned zones (Fig. 6C). These cells were observed in all three experiments, with $n=9,4$, and 5, respectively. Collectively with the data indicating that nearly all cut PCI axons regenerate through the lesion site rather than around the lesion site (compare Fig. 4), nearly all of these antidromic responses may be attributed to the conduction of action potentials by axons that crossed the lesion site. There were no significant differences $(p>0.05)$ (Fig. $6 D)$ between the latencies of antidromic responses recorded from PCIs in all three zones. Data from all of the antidromic experiments were grouped, and the frequency of recording antidromically activated PCIs per electrode track for each of the zones was compared across survival times. We found that there were significantly more antidromically activated PCIs within the lesioned zones of 56-72 d experiments than $0 \mathrm{~d}$ and $7 \mathrm{~d}$ experiments $(p<0.001)$ (Fig. $6 E$ ). We also observed that the lesioned zones with antidromically activated PCIs corresponded to regions with regenerated axons that crossed the lesion site. Together with the survey and reconstruction data, the results shown in this section indicate that regenerated PCI axons conduct action potentials through the lesion site 
and provide a qualitative index of the frequency of axons that had regenerated through the lesion site.

As seen in the $0 \mathrm{~d}$ and $7 \mathrm{~d}$ experiments, in the 56-72 d experiments, antidromically activated PCIs were not detected in many electrode tracts in the lesion zones (Fig. 6C, rostral part of lesion in $72 \mathrm{~d}$ experiment). These regions containing the tracks without antidromically activated PCIs often corresponded to the gray matter regions that were damaged by the midline lesion as described (see above, PCI axons regenerate through the lesion site).

\section{Bouton-like structures on regenerated PCI axons contain synaptophysin}

We next tested whether regenerated PCI axons form putative synaptic connections. Figure 7 compares the distribution of synaptophysin, a synaptic vesicle marker, in boutons of intact and regenerating PCI axons. All of the boutons assigned to the "intact" group arose from reconstructed axons that crossed the midline well rostral or caudal of the lesion site, whereas boutons assigned to the "regenerating" group arose from reconstructed axons that crossed the midline through the lesion site. All of the boutons were located in the ventral horn contralateral to their somata and were examined 56-72 d after injury. Synaptophysin labeling in en passant and terminal boutons on collaterals from uninjured PCIs was restricted to punctate zones (Fig. 7A) (131 boutons were examined in three animals; $n=48,32$, and 51 per animal). Many boutons of regenerated PCI axons also had a similar punctate distribution of synaptophysin (Fig. 7B) (126 boutons were examined in two animals; $n=83$ and 43 per animal); however, a lower proportion of the regenerated boutons contained synaptophysin compared with uninjured boutons (Fig. 7C). There were no differences in the occurrence of synaptophysin-negative boutons examined in different animals. These data indicate that many of the boutons of regenerated PCI axons may be capable of synaptic transmission.

\section{Stimulation of PCI axons evokes PSPs in motoneurons}

We then tested whether regenerated PCI axons evoke PSPs at 56-72 d after injury. Neurons were impaled, and we recorded monosynaptic PSPs evoked by stimuli delivered to regions that contained PCIs in the contralateral cord (Fig. $8 \mathrm{~A}$ ). Data were collected from four of the five animals that were used for reconstructions and from analysis of CSPG and synaptophysin distributions (see previous sections). Data from one animal were excluded because the stim-

$\mathbf{B}$
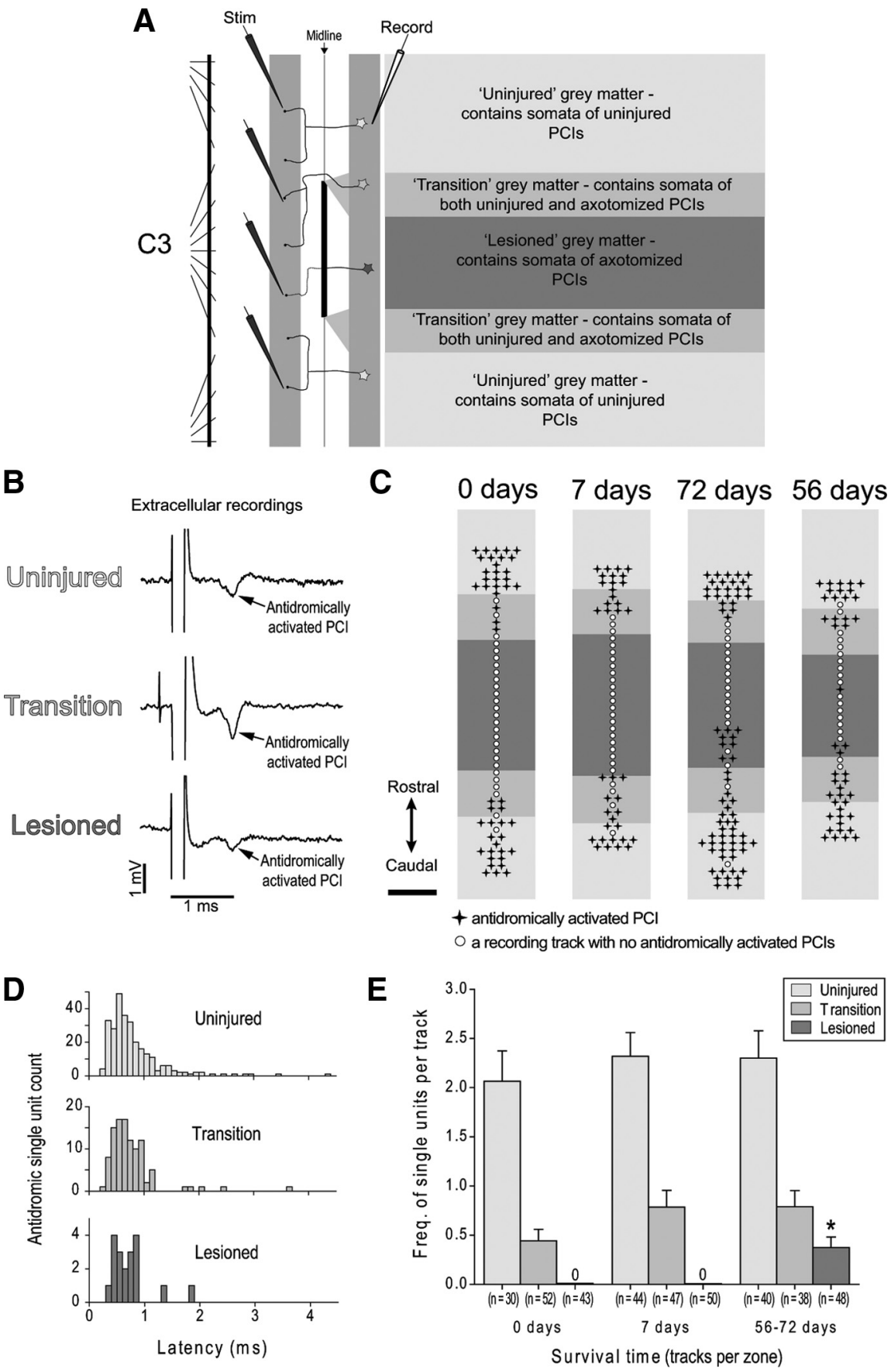

Figure 6. Regenerated $\mathrm{PCl}$ axons conduct action potentials. $\boldsymbol{A}$, Schematic of the experimental setup for recording antidromic responses of stimulated $P C l$ s and locations of uninjured, transition, and lesioned zones. $\boldsymbol{B}$, Representative extracellular recordings of antidromically activated PCls from uninjured, transition, and lesion zones of the gray matter. There is an average of 10 sweeps per trace. C, Representative diagrams of the rostrocaudal distributions of antidromically activated PCIs in the uninjured, transition, and lesioned zones at different postlesion time intervals. Multiple filled symbols arranged in a horizontal row indicate the number of $\mathrm{PCls}$ recorded in the electrode track at that rostrocaudal position. Scale bar, $1 \mathrm{~mm}$. $\boldsymbol{D}$, Histograms showing the latencies of antidromically activated PCls recorded in the uninjured, transition, and lesioned zones. There was no significant difference between the latencies in the uninjured and transition zones at different postlesion times (Kolmogorov-Smirnov test, $p>0.05$ ). These latencies were therefore combined. $\boldsymbol{E}$, Bar graphs showing the average number of antidromically activated PCls recorded per electrode track in the intact, transition, and lesion zones for all 0, 7, and 56-72 d antidromic experiments. Values represent means \pm SEM $\left({ }^{*} p<0.001\right.$, comparison between the 56-72 d lesion zone and 0 and $7 \mathrm{~d}$ lesion zones intervals, using KruskalWallis one-way ANOVA and post hoc analysis using Mann-Whitney $U$ test with Bonferroni correction).

ulating electrodes were discovered to lie outside the lesion zone after histological processing. Similar to the experiments in which antidromically activated PCI action potentials were recorded, the spinal cord was divided into uninjured, transition, and lesioned 

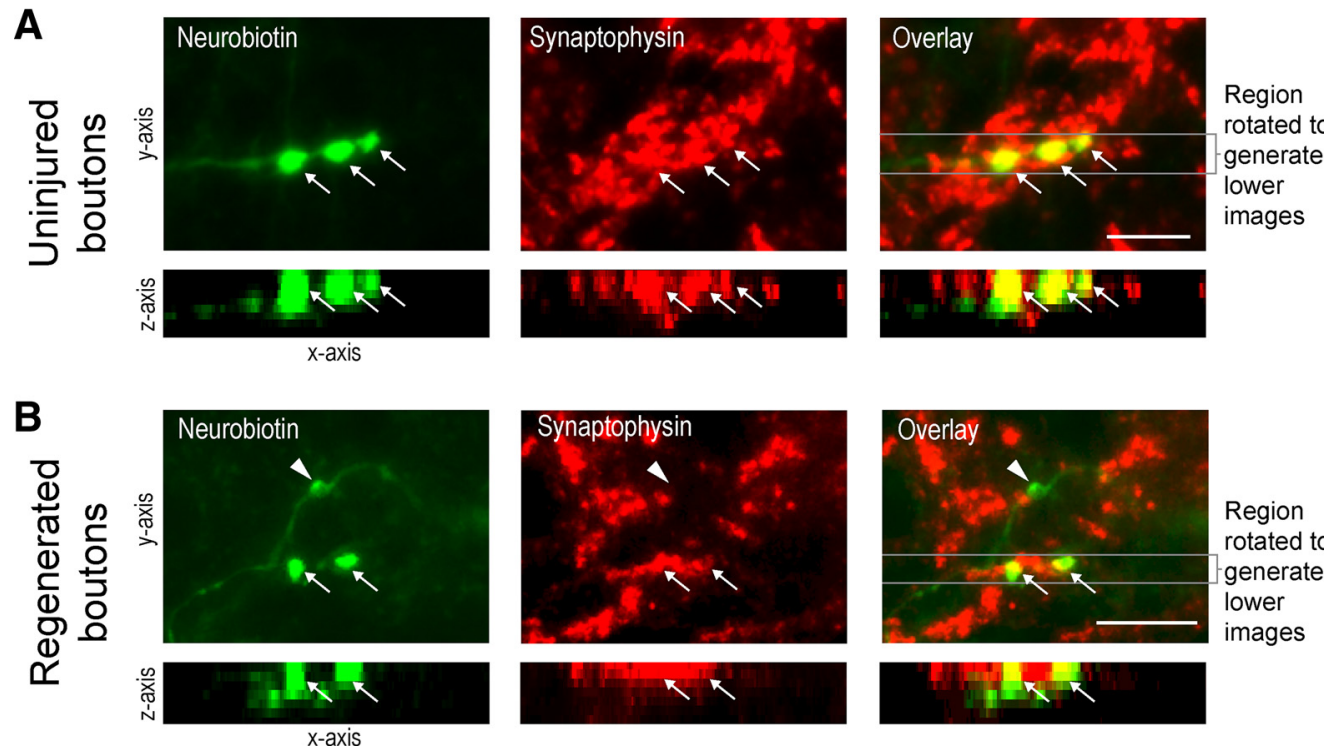

Figure 7. Boutons of regenerated $\mathrm{PCl}$ axons contain synaptophysin. $\boldsymbol{A}, \boldsymbol{B}$, Representative images of boutons from uninjured $(\boldsymbol{A})$ and regenerated $(\boldsymbol{B}) \mathrm{PCl}$ axons that were stained with Neurobiotin and labeled for synaptophysin. Scale bars, $10 \mu \mathrm{m}$. The orientation of the top images is such that rostral is up ( $y$-axis) and medial is right ( $x$-axis). The bottom images were generated by rotating parts of the top images $90^{\circ}$ about the $x$-axis. The regions of the top images that were rotated to generate the bottom images are indicated on the overlays. $A$, Distribution of synaptophysin labeling in three boutons from an intact PCl axon (arrows). All boutons contained synaptophysin. The bottom images consist of nine focal planes ( $z$-axis). Each focal plane is $1 \mu \mathrm{m}$ thick. $\boldsymbol{B}$, Distribution of synaptophysin labeling in boutons from a regenerated PCl axon. Two of the three boutons (indicated by the arrows) contained synaptophysin. Synaptophysin was not visible in the other bouton (indicated by the arrowheads; not shown in the rotated image of the bottom panels). The bottom images consists of seven focal planes ( $z$-axis). Each focal plane is $1 \mu \mathrm{m}$ thick. $C$, Bar graphs showing the proportion of boutons with synaptophysin labeling from intact ( $n=131$ boutons) and regenerated (Regen; $n=126$ boutons) PCl axons.

zones (see above, Regenerated PCI axons conduct action potentials). However, unlike the antidromic experiments, the stimulating electrodes (rather than the recording electrodes) were assigned to one of these zones. As a consequence, the transition zones were lengthened by $250 \mu \mathrm{m}$. This change was designed to take into account the current spread from stimulating electrodes (Stoney et al., 1968). The distance that current spreads from stimulating electrodes is much larger than the spread of extracellularly recorded antidromic action potentials (compare Fig. $6 A, 50 \mu \mathrm{m}$ ).

We recorded from antidromically identified motoneurons, other motoneurons that were not antidromically identified, and interneurons ( $n=37,8$, and 11 , respectively; see Materials and Methods for the criteria of each neuron type). PSPs were evoked after stimulation of the uninjured, transition, and lesioned zones and had latencies ranging from 0.56 to $3.73 \mathrm{~ms}$ (Fig. $8 \mathrm{~B}$ ). The distribution of latencies of PSPs evoked from stimuli delivered to the uninjured zones had two peaks. The midpoint between these peaks corresponded to $1.3 \mathrm{~ms}$. The first peak is a short-latency group and is typically ascribed to monosynaptic connections, the second peak is a long-latency group and is typically ascribed to multisynaptic connections, and the midpoint between the peaks is considered the upper limit for monosynaptic latencies (cf. Sugiuchi et al., 1995). All of these PSPs had a constant latency $<1.3 \mathrm{~ms}$ and reliably followed high-frequency stimulus trains $(>250 \mathrm{~Hz})$.

Monosynaptic PSPs evoked by stimuli delivered to the uninjured and transition zones were recorded in 36 and 25, respectively, of the 58 neurons examined (Fig. 8C). Monosynaptic PSPs evoked by stimulation of the lesioned zones were recorded in six of these neurons (Fig. $8 D)(n=2$ excitatory PSPs; $n=4$ inhibitory PSPs). On an experiment-by-experiment basis, the number of neurons with monosynaptic PSPs evoked by stimuli in the lesioned zones was 2, 3, 1, and 0 . All neurons that received monosynaptic input from PCIs in the lesioned zones were motoneurons $(n=4$ antidromically identified motoneurons; $n=2$ motoneurons not antidromically identified). These motoneurons also received monosynaptic and/or multisynaptic connections from PCIs in the transition and intact zones.

The probability of evoking a monosynaptic PSP was calculated for each of the stimulus zones (Fig. 8E). To account for differences between the number of stimulating electrodes for any given zone (compare Fig. $8 \mathrm{~A}$ ), the probability of recording a monosynaptic PSP was weighted by the number of stimulating electrodes in each region. We found that the probability of evoking PSPs was highest after stimulation of the uninjured zone, lowest after stimulation of the lesioned zone, and intermediate after stimulation of the transition zone. This pattern is consistent with the decreasing frequency of antidromically activated PCIs in the respective zones for the 56-72 d experiments (Fig. 6F). Together, these data indicate that some motoneurons receive monosynaptic connections from regenerated PCIs and that regenerated PCIs form connections with neurons that typically receive connections from uninjured PCIs.

\section{Discussion}

After SCI, the spontaneous sprouting of spinal interneuron axons plays an important role in restoring function by increasing synaptic connections within existing spinal circuits and forming new spinal circuits around the injury site (Bareyre et al., 2004; Courtine et al., 2008). Here, we show that spinal interneuron axons are also capable of spontaneously regenerating through the inhibitory environment of the injury site and forming functional synaptic connections with appropriate neural targets.

\section{Regeneration despite inhibition}

To our knowledge, this is the first study to describe a class of adult mammalian CNS neurons that can spontaneously regenerate through an environment that would typically be considered highly inhibitory (i.e., contains CSPGs). This raises the question, What allows PCI axons to grow in an environment where all 
other known CNS axons fail to grow? There are several strategies PCI axons may use to grow despite a close interaction with an inhibitory environment.

First, it is possible that PCI axons are not inhibited by inhibitory molecules. This is conceivable since some populations of axons are better than others at growing in inhibitory environments (Snow and Letourneau, 1992; Inman and Steward, 2003). Inhibitory molecules prevent growth cone progression primarily through activation of the RhoA-ROCK pathway (Winton et al., 2002; Monnier et al., 2003; Yiu and He, 2006; Conrad et al., 2007), which disrupts the regulation of the actin cytoskeleton (Maekawa et al., 1999). It is therefore possible that the RhoA-ROCK pathway is not activated in regenerating PCI axons in response to inhibitory environments.

It is also possible that PCI axons may have an unusual capacity for growth, which supersedes the inhibitory effects of CSPGs. Proximal axotomies are linked to altered expression of growth-associated proteins such as GAP-43, $\alpha$-tubulins, and neurofilament-m (Fernandes et al., 1999) and are associated with increased axonal growth capacity(Richardson et al., 1984). We have previously shown that PCIs are proximally axotomized and express GAP-43 after a midline lesion (Fenrich et al., 2007). GAP-43 expression is often used as an indicator of axonal regenerative capacity and is associated with actin stabilization within growth cones (Denny, 2006). These data support the idea that proximally axotomized PCI axons may overcome an inhibitory environment.

Another strategy may be that PCI growth cones are highly adept at "path finding." In vitro, adult growth cones have dynamic structures that protrude and retract along gradients of CSPGs (Tom et al., 2004). At 7 d after lesion, the distribution of CSPGs at the lesion site is not uniform, and PCI growth cones tend to have multiple branches, varicosities, and tortuous trajectories. Many of the branches of PCI growth cones are in close apposition with dense CSPG labeling, whereas other branches are within zones of relatively low CSPG labeling. Given this arrangement, it is possible that PCI growth cones extend multiple branches to seek out regions with less inhibitory molecules. Branches that project to regions of little inhibition remain viable, whereas branches that extend to regions of dense inhibition collapse and retract. Repetition of this process would allow growth cones to extend beyond highly inhibitory regions of the injury site.
A

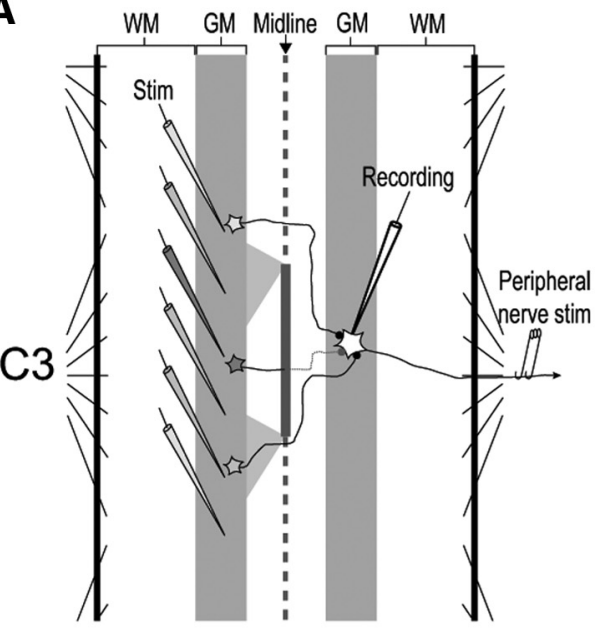

B
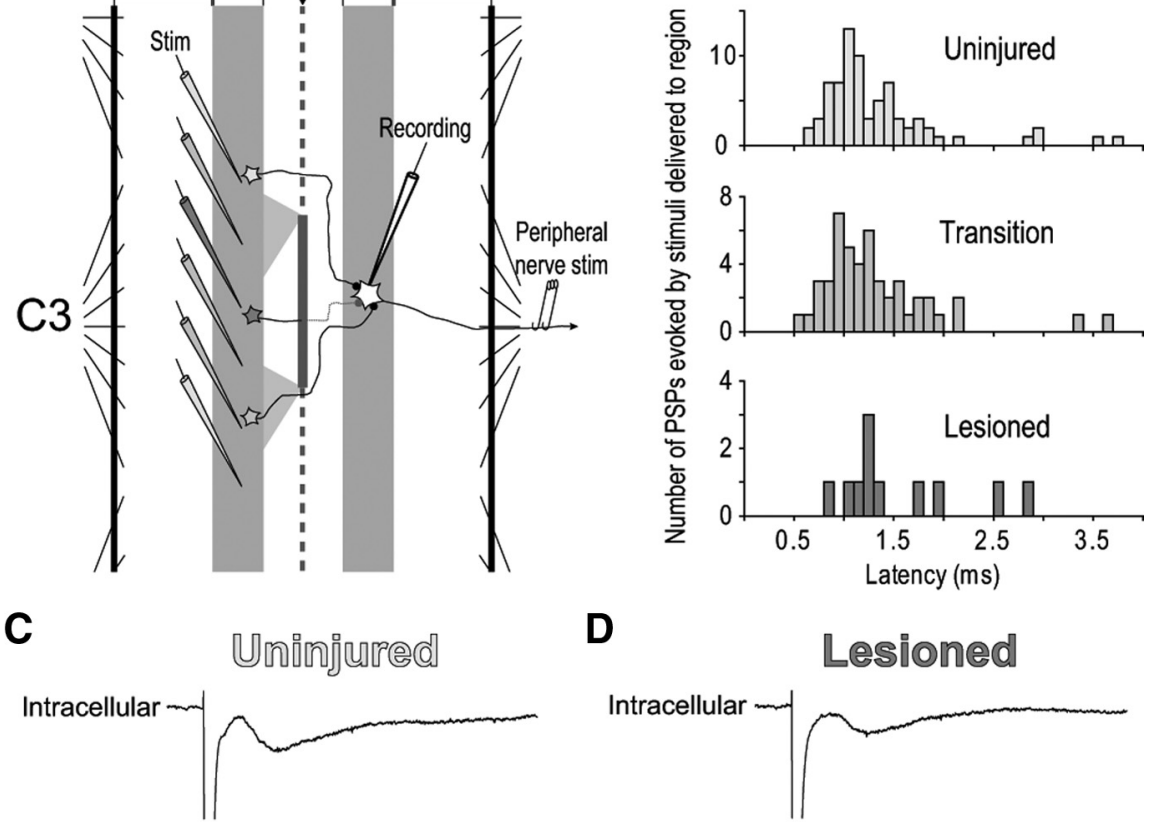

D Lesioned
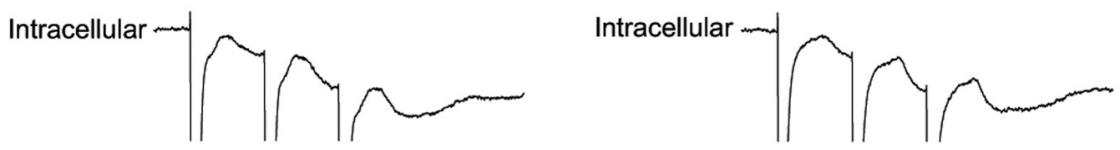

Extracellular n- $\| \int_{1 \mathrm{~ms}} \stackrel{\text { हो }}{-}$

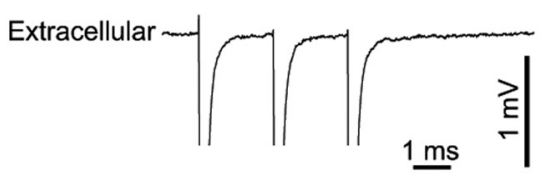

E

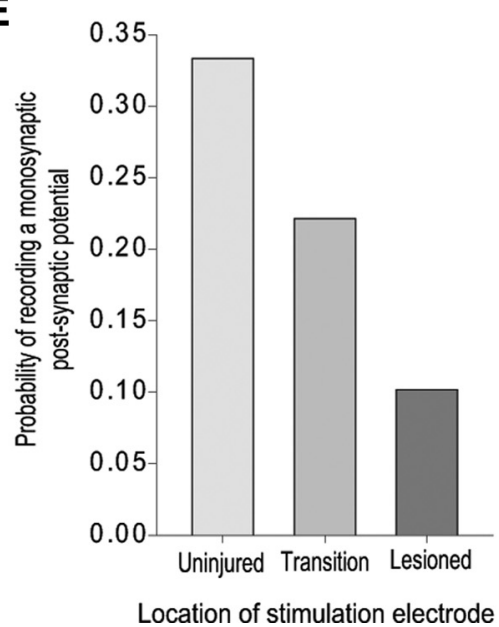

Figure 8. Regenerated PCl axons form functional synaptic connections. A, Schematic of the position of stimulating (Stim) electrodes relative to the lesion and intracellular recordings. The stimulating electrodes are colored according to the zone in which they are located: light gray, uninjured zones; medium gray, transition zones; dark gray, lesioned zones. $\boldsymbol{B}$, Histograms showing the latencies of PSPs evoked by stimuli delivered to uninjured, transition, and lesioned zones. $\boldsymbol{C}, \boldsymbol{D}$, Representative examples of monosynaptic PSPs recorded in motoneurons, in response to stimulation of the uninjured $(\boldsymbol{C})$ and lesioned $(\boldsymbol{D})$ zones. Top traces show inhibitory monosynaptic PSPs in response to a single stimulus. Middle traces show PSPs in response to three consecutive stimuli $(500 \mathrm{~Hz})$ delivered to the same electrode used to evoke the responses shown in the top traces. Bottom traces show the responses recorded extracellularly to the motoneuron somata (same stimulus parameters as for the middle traces). There is an average of 25 sweeps per trace. $E$, Bar graph showing the probability of evoking a PSP from stimuli delivered to the uninjured, transition, and lesioned zones. The probabilities were weighted to account for the number of stimulating electrodes in each zone for each experiment. WM, White matter; GM, gray matter. 
To gain an understanding of how PCI axons regenerate, it will be vital to study whether PCI axons use any one or a combination of these strategies to navigate the inhibitory environment of the injury site. In particular, a description of how the molecular pathways associated with growth cone extension and collapse are affected and controlled in PCI axons is a crucial step for understanding how PCI axons react to inhibitory environments. Also, since some PCI axons did not regenerate through the lesion site, to fully understand PCI axon regeneration, it is important to determine what advantage some PCI axons have for regenerating over those that do not regenerate.

\section{Crossing the midline}

Guidance of spinal axons across the midline is one of the most complex and extensively studied processes of neural development. Our data show that, despite many potential neural targets ipsilateral of the lesion site, regenerated PCI axons cross the lesioned midline to form synaptic connections with contralateral neurons. This implies that regenerating PCI axons are guided toward and across the lesioned midline.

During development, spinal axons that cross the midline are initially attracted to guidance proteins released at the ventral midline, but these axons switch their attraction to repulsion once they have crossed the midline; this switch promotes growth beyond the midline and prevents recrossing the midline. Guidance proteins from the midline include netrin-1 (Serafini et al., 1996), slits (Brose et al., 1999; Kidd et al., 1999), and Sonic hedgehog (Shh) (Charron et al., 2003). All of these guidance proteins are upregulated at or near the injury site after SCI (Chen et al., 2005; Wehrle et al., 2005). Together, it is tempting to speculate that lesions restricted to the midline lead to the release of guidance molecules from within the lesion site, thus mimicking the mediolateral distribution of these molecules during development.

When presented with an appropriate distribution of guidance molecules at the midline, axons must first be attracted toward then repelled by these guidance molecules to cross the midline. Before crossing, developing axons express the receptors DCC (Deleted in colorectal cancer) (KeinoMasu et al., 1996), Robo3.1 (Chen et al., 2008), and Boc (biregional Cdon-binding protein) (Okada et al., 2006), which are known to attract the axons toward netrin-1, slit, and Shh, respectively. At the midline, developing axons express the "repulsion" receptors UNC-5 (Hong et al., 1999), Robo3.2 and Robo1/2 (Chen et al., 2008), and Hip (Bourikas et al., 2005), which leads to repulsion of developing axons away from netrin-1, slit, and Shh, respectively. Our data shows that cut PCI axons recapitulate their original growth trajectories by regenerating across the midline. However, after axotomy, axons that are typically restricted to one side of the spinal cord can also sprout new collaterals that cross midline (Bareyre et al., 2004; Courtine et al., 2008). This suggests that there may be an inherent ability for certain populations of cut axons to respond to guidance cues and cross the midline. Low et al. (2008) have shown that cut rubrospinal axons respond to netrin-1 after SCI. However, the study by Low et al. (2008) showed that netrin-1 inhibits rubrospinal axon growth rather than guide axons across the midline. Together, these data indicate that, in response to injury, some axons may respond to guidance molecules in a way that allows them to cross the midline, whereas others are inhibited by these molecules.

\section{Synaptic connections with appropriate targets}

Our data shows that regenerated PCI axons have collaterals that project to the contralateral ventral gray matter, similar to intact
PCI axons. In addition, we found that regenerated PCI axons form synaptic connections with motoneurons that receive input from intact PCI axons (Sugiuchi et al., 1992, 1995). Although we cannot preclude the possibility that some regenerated PCI axons form aberrant connections, our results indicate that regenerating PCI axons form connections with appropriate targets once they have crossed the lesion site. How PCIs are guided to their appropriate targets and form connections with these targets is unknown. One possible mechanism is by forming surplus connections and retaining only those that are functionally appropriate. For instance, in response to partial spinal lesions, spinal interneurons form a surplus of new synaptic connections within a few weeks of injury, but many of these new connections retract within several weeks of their formation, and only the connections that contribute to functional recovery remain (Bareyre et al., 2004). It has been speculated that the functionally relevant connections are maintained through activity-dependent stabilization of appropriate connections (Bareyre et al., 2004). It is possible that surplus connections are formed by regenerating PCI axons and these connections are pruned over time. Currently, we cannot determine whether significant pruning has occurred, since this study has only one postaxotomy interval with collaterals from regenerated PCI axons $(56-72 \mathrm{~d})$.

\section{Significance for spinal injuries}

Previously it was believed that adult mammalian CNS axons were incapable of regeneration through an inhibitory injury site in the absence of therapeutic interventions. Our results show that not all regenerative attempts after CNS lesions are futile and that at least some spinal interneurons are capable of spontaneous functional regeneration across inhibitory lesions. After partial SCIs, in addition to the plasticity of their axons, spinal interneurons also receive new connections from axotomized corticospinal axons. Spinal interneurons thus act as "relays" for injured corticospinal signals around spinal lesions (Bareyre et al., 2004; Courtine et al., 2008). Although we could not test whether PCIs with regenerated axons receive new inputs after a midline lesion, one might speculate that they may also serve as relays through spinal lesions by receiving new inputs from cut axons that cannot regenerate.

This study shows that spinal commissural interneurons can regenerate after SCI. Resolving the molecular mechanisms that allow PCI axons to grow through an inhibitory environment and to find appropriate targets will prove an important tool for devising new therapeutic strategies for enhancing regeneration of other descending and ascending spinal axons after SCIs.

\section{References}

Bareyre FM, Kerschensteiner M, Raineteau O, Mettenleiter TC, Weinmann O, Schwab ME (2004) The injured spinal cord spontaneously forms a new intraspinal circuit in adult rats. Nat Neurosci 7:269-277.

Bolton PS, Goto T, Wilson VJ (1991) Commissural neurons in the cat upper cervical spinal cord. Neuroreport 2:743-746.

Bourikas D, Pekarik V, Baeriswyl T, Grunditz A, Sadhu R, Nardo M, Stoeckli ET (2005) Sonic hedgehog guides commissural axons along the longitudinal axis of the spinal cord. Nat Neurosci 8:297-304.

Bradbury EJ, Moon LD, Popat RJ, King VR, Bennett GS, Patel PN, Fawcett JW, McMahon SB (2002) Chondroitinase ABC promotes functional recovery after spinal cord injury. Nature 416:636-640.

Bregman BS, Kunkel-Bagden E, Schnell L, Dai HN, Gao D, Schwab ME (1995) Recovery from spinal cord injury mediated by antibodies to neurite growth inhibitors. Nature 378:498-501.

Brose K, Bland KS, Wang KH, Arnott D, Henzel W, Goodman CS, TessierLavigne M, Kidd T (1999) Slit proteins bind robe receptors and have an evolutionarily conserved role in repulsive axon guidance. Cell 96:795-806. 
Charron F, Stein E, Jeong J, McMahon AP, Tessier-Lavigne M (2003) The morphogen Sonic hedgehog is an axonal chemoattractant that collaborates with Netrin-1 in midline axon guidance. Cell 113:11-23.

Chen J, Leong SY, Schachner M (2005) Differential expression of cell fate determinants in neurons and glial cells of adult mouse spinal cord after compression injury. Eur J Neurosci 22:1895-1906.

Chen Z, Gore BB, Long H, Ma L, Tessier-Lavigne M (2008) Alternative splicing of the Robo3 axon guidance receptor governs the midline switch from attraction to repulsion. Neuron 58:325-332.

Conrad S, Genth H, Hofmann F, Just I, Skutella T (2007) Neogenin-RGMa signaling at the growth cone is bone morphogenetic protein-independent and involves RhoA, ROCK, and PKC. J Biol Chem 282:16423-16433.

Conta AC, Stelzner DJ (2004) Differential vulnerability of propriospinal tract neurons to spinal cord contusion injury. J Comp Neurol 479:347359.

Courtine G, Song BB, Roy RR, Zhong H, Herrmann JE, Ao Y, Qi JW, Edgerton VR, Sofroniew MV (2008) Recovery of supraspinal control of stepping via indirect propriospinal relay connections after spinal cord injury. Nat Med 14:69-74.

Davies SJ, Goucher DR, Doller C, Silver J (1999) Robust regeneration of adult sensory axons in degenerating white matter of the adult rat spinal cord. J Neurosci 19:5810-5822.

Denny JB (2006) Molecular mechanisms, biological actions, and neuropharmacology of the growth-associated protein GAP-43. Curr Neuropharmacol 4:293-304.

Fenrich KK, Skelton N, MacDermid VE, Meehan CF, Armstrong S, NeuberHess MS, Rose PK (2007) Axonal regeneration and development of de novo axons from distal dendrites of adult feline commissural interneurons after a proximal axotomy. J Comp Neurol 502:1079-1097.

Fernandes KJ, Fan DP, Tsui BJ, Cassar SL, Tetzlaff W (1999) Influence of the axotomy to cell body distance in rat rubrospinal and spinal motoneurons: differential regulation of GAP-43, tubulins, and neurofilament-M. J Comp Neurol 414:495-510.

Grande G, Armstrong S, Neuber-Hess M, Rose PK (2005) Distribution of contacts from vestibulospinal axons on the dendrites of splenius motoneurons. J Comp Neurol 491:339-351.

Harel NY, Strittmatter SM (2006) Can regenerating axons recapitulate developmental guidance during recovery from spinal cord injury? Nat Rev Neurosci 7:603-616.

Hong KS, Hinck L, Nishiyama M, Poo MM, Tessier-Lavigne M, Stein E (1999) A ligand-gated association between cytoplasmic domains of UNC5 and DCC family receptors converts netrin-induced growth cone attraction to repulsion. Cell 97:927-941.

Inman DM, Steward O (2003) Ascending sensory, but not other long-tract axons, regenerate into the connective tissue matrix that forms at the site of a spinal cord injury in mice. J Comp Neurol 462:431-449.

KeinoMasu K, Masu M, Hinck L, Leonardo ED, Chan SSY, Culotti JG, Tessier-Lavigne M (1996) Deleted in Colorectal Cancer (DCC) encodes a netrin receptor. Cell 87:175-185.

Kidd T, Bland KS, Goodman CS (1999) Slit is the midline repellent for the robe receptor in Drosophila. Cell 96:785-794.

Low K, Culbertson M, Bradke F, Tessier-Lavigne M, Tuszynski MH (2008) Netrin-1 is a novel myelin-associated inhibitor to axon growth. J Neurosci 28:1099-1108.

Lu P, Yang H, Jones LL, Filbin MT, Tuszynski MH (2004) Combinatorial therapy with neurotrophins and cAMP promotes axonal regeneration beyond sites of spinal cord injury. J Neurosci 24:6402-6409.

Maekawa M, Ishizaki T, Boku S, Watanabe N, Fujita A, Iwamatsu A, Obinata T, Ohashi K, Mizuno K, Narumiya S (1999) Signaling from rho to the actin cytoskeleton through protein kinases ROCK and LIM-kinase. Science 285:895-898.
McKeon RJ, Schreiber RC, Rudge JS, Silver J (1991) Reduction of neurite outgrowth in a model of glial scarring following CNS injury is correlated with the expression of inhibitory molecules on reactive astrocytes. J Neurosci 11:3398-3411.

Monnier PP, Sierra A, Schwab JM, Henke-Fahle S, Mueller BK (2003) The Rho/ROCK pathway mediates neurite growth-inhibitory activity associated with the chondroitin sulfate proteoglycans of the CNS glial scar. Mol Cell Neurosci 22:319-330.

Neumann S, WoolfCJ (1999) Regeneration of dorsal column fibers into and beyond the lesion site following adult spinal cord injury. Neuron 23: 83-91.

Okada A, Charron F, Morin S, Shin DS, Wong K, Fabre PJ, Tessier-Lavigne M, McConnell SK (2006) Boc is a receptor for sonic hedgehog in the guidance of commissural axons. Nature 444:369-373.

Plunet W, Kwon BK, Tetzlaff W (2002) Promoting axonal regeneration in the central nervous system by enhancing the cell body response to axotomy. J Neurosci Res 68:1-6.

Ramon y Cajal S (1959) Degeneration and regenerations of the nervous system. New York: Hafner.

Richardson PM, Issa VM, Aguayo AJ (1984) Regeneration of long spinal axons in the rat. J Neurocytol 13:165-182.

Richardson PM, Issa VMK (1984) Peripheral injury enhances central regeneration of primary sensory neurons. Nature 309:791-793.

Schnell L, Schwab ME (1990) Axonal regeneration in the rat spinal cord produced by an antibody against myelin-associated neurite growth inhibitors. Nature 343:269-272.

Serafini T, Colamarino SA, Leonardo ED, Wang H, Beddington R, Skarnes WC, Tessier-Lavigne M (1996) Netrin-1 is required for commissural axon guidance in the developing vertebrate nervous system. Cell 87:10011014.

Shinoda Y, Arnold AP, Asanuma H (1976) Spinal branching of corticospinal axons in cat. Exp Brain Res 26:215-234.

Silver J, Miller JH (2004) Regeneration beyond the glial scar. Nat Rev Neurosci 5:146-156.

Snow DM, Letourneau PC (1992) Neurite outgrowth on a step gradient of chondroitin sulfate proteoglycan (Cs-Pg). J Neurobiol 23:322-336.

Steward O, Zheng B, Tessier-Lavigne M (2003) False resurrections: distinguishing regenerated from spared axons in the injured central nervous system. J Comp Neurol 459:1-8.

Stoney SD, Thompson WD, Asanuma H (1968) Excitation of pyramidal tract cells by intracortical microstimulation-effective extent of stimulating current. J Neurophysiol 31:659-669.

Sugiuchi Y, Kakei S, Shinoda Y (1992) Spinal commissural neurons mediating vestibular input to neck motoneurons in the cat upper cervical spinal-cord. Neurosci Lett 145:221-224.

Sugiuchi Y, Izawa Y, Shinoda Y (1995) Trisynaptic inhibition from the contralateral vertical semicircular canal nerves to neck motoneurons mediated by spinal commissural neurons. J Neurophysiol 73:1973-1987.

Tom VJ, Steinmetz MP, Miller JH, Doller CM, Silver J (2004) Studies on the development and behavior of the dystrophic growth cone, the hallmark of regeneration failure, in an in vitro model of the glial scar and after spinal cord injury. J Neurosci 24:6531-6539.

Wehrle R, Camand E, Chedotal A, Sotelo C, Dusart I (2005) Expression of netrin-1, slit-1 and slit-3 but not of slit-2 after cerebellar and spinal cord lesions. Eur J Neurosci 22:2134-2144.

Winton MJ, Dubreuil CI, Lasko D, Leclerc N, McKerracher L (2002) Characterization of new cell permeable C3-like proteins that inactivate Rho and stimulate neurite outgrowth on inhibitory substrates. J Biol Chem 277:32820-32829.

Yiu G, He Z (2006) Glial inhibition of CNS axon regeneration. Nat Rev Neurosci 7:617-627. 